\title{
Microfluidics for pharmaceutical nanoparticle fabrication: the truth and the myth
}

Sherif I. Hamdallah ${ }^{1,2}$, Randa Zoqlam ${ }^{1}$, Peer Erfle ${ }^{3,4}$, Mark Blyth ${ }^{5}$, Alaaldin M. Alkilany. A. M. ${ }^{6}$, Andreas Dietzel ${ }^{3,4}$, Sheng $\mathrm{Qi}^{1}$

${ }^{1}$ School of Pharmacy, University of East Anglia, Norwich, NR4 7TJ, UK

${ }^{2}$ Department of Pharmaceutics, Faculty of Pharmacy, Alexandria University, Alexandria, Egypt

${ }^{3}$ Technische Universität Braunschweig, Institut für Mikrotechnik / Institute of Microtechnology, Alte Salzdahlumer Str. 203, Geb. 1A, 38124 Braunschweig

${ }^{4}$ Technische Universität Braunschweig, Center of Pharmaceutical Engineering, Franz-LisztStr. 35a, 38106 Braunschweig, Germany

${ }^{5}$ School of Mathematics, University of East Anglia, Norwich, NR4 7TJ, UK

${ }^{6}$ Department of Pharmaceutics \& Pharmaceutical Technology, School of Pharmacy, The University of Jordan, Amman, 11942, Jordan

Corresponding author: Sheng Qi, sheng.qi@uea.ac.uk 


\begin{abstract}
Using micro-sized channels to manipulate fluids is the essence of microfluidics which has wide applications from analytical chemistry to material science and cell biology research. Recently, using microfluidic-based devices for pharmaceutical research, in particular for the fabrication of micro- and nano-particles, has emerged as a new area of interest. The particles that can be prepared by microfluidic devices can range from micron size droplet-based emulsions to nano-sized drug loaded polymeric particles. Microfluidic technology poses unique advantages in terms of the high precision of the mixing regimes and control of fluids involved in formulation preparation. As a result of this, monodispersity of the particles prepared by microfluidics is often recognised as being a particularly advantageous feature in comparison to those prepared by conventional large-scale mixing methods. However, there is a range of practical drawbacks and challenges of using microfluidics as a direct micron- and nano-particle manufacturing method. Technological advances are still required before this type of processing can be translated for application by the pharmaceutical industry. This review focuses specifically on the application of microfluidics for pharmaceutical solid nanoparticle preparation and discusses the theoretical foundation of using the nanoprecipitation principle to generate particles and how this is translated into microfluidic design and operation.
\end{abstract}

Keywords: nanoparticles, microfluidics, nanoprecipitation, nucleation, growth, nanocrystals, particle size, microchip design 


\section{Introduction}

Microfluidics concerns the design and fabrication of miniaturized microscale devices containing channels and chambers of different geometry in which fluid mixing can be controlled precisely (Nguyen et al., 2013; Ong et al., 2008; Tsui et al., 2013; Whitesides, 2006). In contrast to macroscale flow systems, where inertial forces play an important role, viscous forces dominate in microfluidic devices, and this explains why laminar flow is typical in these miniaturized systems (Ahn et al., 2018; Liu et al., 2015; Lu et al., 2016; Riahi et al., 2015). Microfluidics technology is a multidisciplinary research field that brings together technologies and principles from fluid dynamics, physics, biology, microtechnology, material science and chemistry. The point of merger of analytical chemistry and biochemistry with microelectronics is considered the real birth of microfluidic technology (Tian and Finehout, 2009). Microfluidics technology has found extensive applications in various areas of science. Applications to pharmaceutical technologies include fabricating nanoparticle-based drug delivery systems (Damiati et al., 2018; Lu et al., 2016; Martins et al., 2018; Pan et al., 2017; Weigl et al., 2003) and performing pre-clinical tests for substances and formulations using the so-called "organ-on-chip" systems (Beißner et al., 2016; Bhise et al., 2014; Li and Tian, 2018). There is a vast number of excellent reviews on both topics (Liu et al., 2018; Martins et al., 2018; Probst et al., 2018; Riahi et al., 2015; Valencia et al., 2012). The emphasis of the present review is on discussing the advantages and disadvantages of using microfluidics for the fabrication of polymeric nanoparticles and drug nanosuspensions via the nanoprecipitation method.

For pharmaceutical applications, the functionality of the nanoparticles is often determined by key characteristics such as their shape, size, and surface properties (Ealias and Saravanakumar, 2017; Jeevanandam et al., 2018; Pande and Ashok Bhaskarwar, 2016). The 
desired properties of nanoparticles can be achieved with an appropriate method of preparation. There are two approaches for nanoparticle formation, namely the top-down and bottom-up approaches (Jeevanandam et al., 2018; Pande and Ashok Bhaskarwar, 2016). Topdown approaches involve particle size reduction by comminution (usually a high-energy process, for example ball milling). Bottom-up techniques are commonly used for the synthesis of organic nanoparticles, in which the material (drug and/or polymer) is dissolved in an appropriate solvent and then thoroughly mixed with an antisolvent to induce particle formation via nucleation, growth and precipitation. This approach builds nanostructured materials gradually from molecules or atoms into larger structures of nanometer size through a controlled precipitation process (Pande and Ashok Bhaskarwar, 2016). The difference between the various methods that follow this approach is the mechanism used to induce the phase separation (precipitation) of the required component (Pande and Ashok Bhaskarwar, 2016). Phase separation can be induced by many factors such as the ambient temperature and $\mathrm{pH}$, and by a change in the solubility of the components. Therefore a fuller understanding of the phase separation mechanism is key to understanding how to control precipitation and to obtaining the desired nanoparticles (Pande and Ashok Bhaskarwar, 2016).

This review has a particular focus on antisolvent based nanoprecipitation using microfluidic devices for the fabrication of polymeric and drug nanoparticles. Traditionally, nanoprecipitation can be achieved by mixing bulk volumes of the solvent (containing the solute) and the antisolvent using conventional mixing methods. The unique advantage of microfluidic systems compared to conventional bulk mixing methods is their ability to offer precise controllability and tunability of the mixing process (Marre and Jensen, 2010; Martins et al., 2018; Su et al., 2007) that, in principle, can be scaled up by parallelized systems (Lim et al., 2014a). This review aims first to provide an overview of the results that can be 
achieved by using microfluidic mixing in the nanoprecipitation process of either polymeric or drug nanoparticles. Second, it aims to assess the pros and cons of using microfluidics in nanoprecipitation compared to other conventional bulk mixing methods that have advantages in high throughput.

\section{The Theory: Nucleation and growth of nanoparticles by nanoprecipitation}

\subsection{Thermodynamics of homogeneous nucleation}

Nucleation is the process that precedes the formation of a new phase due to a change in the system equilibrium (De Yoreo, 2003; Dimo Kashchiev, 2000; Vekilov, 2010; Xiang Y. Liu, 2001). It can be induced by a change in the solute concentration to exceed the equilibrium solubility (supersaturation) or by a drop of the system temperature below the phase transformation point (supercooling). Supercooled or supersaturated systems possess high

Gibbs free energy $(\Delta G)$, which gets reduced by the formation of molecular or atomic clusters (Dimo Kashchiev, 2000). Part of these clusters form the nuclei and exist in equilibrium with the dissolved parent phase. Only super-nuclei, which are nuclei that reach the critical size, can proceed to grow, while nuclei that are below the critical dimensions dissociate in the dissolved parent phase. Nucleation that occurs spontaneously is known as homogeneous nucleation. It describes systems where nuclei are formed uniformly throughout the system. However, nucleation that can be induced by the presence of foreign entities that form a nucleating surface such as impurities, dust or the rough surfaces of the container is known as heterogeneous nucleation (Sear, 2006; Thanh et al., 2014). In the most reported cases of using the nanoprecipitation method to produce nanoparticles, the underpinning mechanism is dominated by homogeneous nucleation. Therefore, in this review we exclusively focus the discussion on the factors controlling the homogeneous nucleation rate and their subsequent effects on the properties of the nanoparticles. 
The nucleation process is associated with a change in the total Gibbs free energy $(\Delta G)$ of the system, which can be interpreted as the sum of the excess surface free energy change per unit area $(\Delta \mu s)$ and the excess volume free energy change $(\Delta G v)$ per unit volume $(\Delta \mu v)$, as shown in Eq. 1 (Ealias and Saravanakumar, 2017; Polte, 2015; Thanh et al., 2014; Liu, 2001). Assuming the formation of a spherical nucleus ( $r$ as the radius of the spherical nucleus), $\Delta \mu s$, $\Delta \mu \nu$, surface energy $(\gamma)$ and the total free energy change can be rearranged as shown in Eq. 2-4 (Ealias and Saravanakumar, 2017; Polte, 2015; Thanh et al., 2014; Liu, 2001).

$\Delta G=\Delta \mu v+\Delta \mu s$

$\Delta \mu s=4 \pi r^{2} \gamma$

Eq. 2

$\Delta \mu v=(4 / 3) \pi r^{3} \Delta G v$

Eq. 3

$\Delta G=\left(\frac{4}{3}\right) \pi r^{3} \Delta G v+4 \pi r^{2} \gamma$

Eq. 4

Spontaneous nucleation is driven by the reduction of the overall Gibbs free energy of a supersaturated solution due to the formation of a solid phase (the precipitated particles which provide the excess volume and surface within the system) and the maintenance of an equilibrium concentration in the solution (Cao and Wang, 2011). This reduction is attributable to the negative value of $\Delta G v$, which is dependent on the solute concentration, emphasizing that supersaturation is the main driving force for nucleation as illustrated in Eq. 5 (Dimo Kashchiev, 2000), where $\Delta G v$ is the free energy of the bulk crystal, $k$ is Boltzmann's constant, $T$ is the absolute temperature (in Kelvin), $C$ is the solute concentration, $C o$ is the solubility, $\Omega$ is the atomic volume and $\sigma$ is the degree of supersaturation that can be defined as $(C-C o) / C o$. When the system is undersaturated $(\sigma=0)$ and $\Delta G \nu=0$, nucleation cannot take place. However, when the system is supersaturated (i.e. $C>C o$ ), $\Delta G v$ is negative and spontaneous nucleation predominates (Figure 1).

$$
\Delta G v=-\frac{k T}{\Omega} \ln \left(\frac{C}{C o}\right)=-\frac{k T}{\Omega} \ln (1+\sigma) \quad \text { Eq. } 5
$$




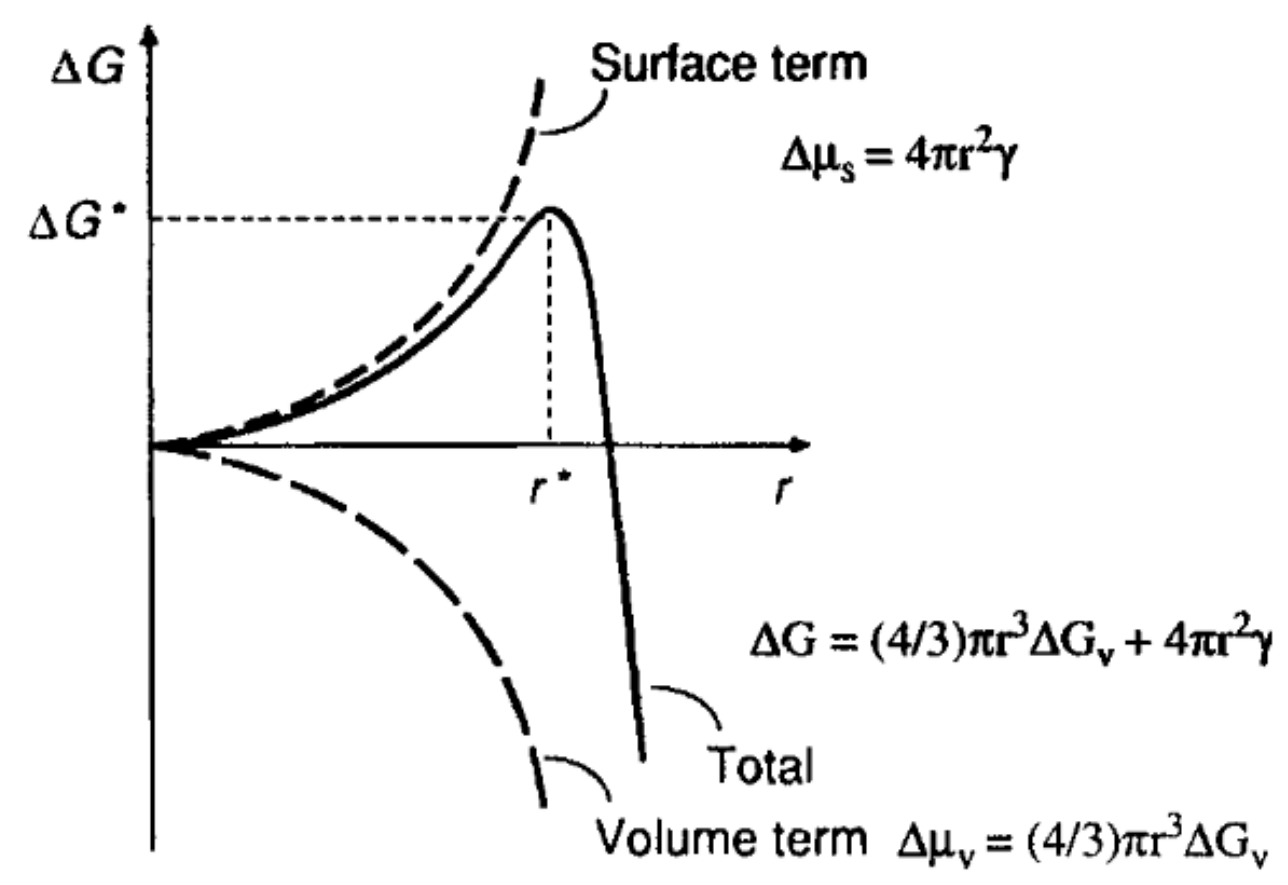

Figure 1. Schematic illustration of the total free energy change as a function of nucleus radius (reprinted with permission from (Thanh et al., 2014)).

The reduction in the total Gibbs free energy is compensated by an increase in the surface energy by an amount equal to the interfacial tension for each unit area of the forming interface. Consequently, the net free energy of nucleation can be interpreted as the sum of the surface free energy and the volume free energy as illustrated by Eq. 4 (Ealias and Saravanakumar, 2017; Polte, 2015; Thanh et al., 2014; Liu, 2001). The overall free energy change increases with the size increase until the nucleus grows to the maximum size defined as the critical size $\left(r^{*}\right)$ (Figure 1). After this, the net free energy undergoes a gradual decrease for any further increase in the nucleus size (Polte, 2015; Thanh et al., 2014). This is known as the LaMer mechanism, which states that nucleation and growth of nanoparticles occur in three stages (Thanh et al., 2014). The first stage is characterized by an increase in the solute concentration to reach the minimum solute concentration needed to induce nucleation 
(referred as $C_{\min }$ ) (Thanh et al., 2014). This is followed by the next stage, where "burst nucleation" takes place leading to a significant reduction in the solute concentration, which consequently reduces the nucleation rate to zero (Thanh et al., 2014). The last stage is known to be a diffusion-controlled growth process. The minimum size needed for the particle to proceed to the growth phase is known as the critical radius, $r^{*}$ (Thanh et al., 2014). Two important terms that can be derived from Eq. 4, which are the critical radius $\left(r^{*}\right)$ and critical $\Delta G\left(\Delta G^{*}\right)$, are expressed in Eq. 6 and Eq.7 (Polte, 2015; Thanh et al., 2014; Xiang Y. Liu, 2001).

$$
r^{*}=-2 \gamma / \Delta G v \quad \text { Eq. } 6
$$

Critical $\Delta \mathrm{G}\left(\Delta \mathrm{G}^{*}\right)$ illustrated in Figure $\mathbf{1}$ is defined as the energy barrier that must be overcome to obtain a stable nucleus (Polte, 2015; Thanh et al., 2014; Xiang Y. Liu, 2001):

$\Delta G^{*}=\frac{16 \pi \gamma}{(3 \Delta G v)^{2}} \quad$ Eq. 7

In conclusion, these equations explain that the critical size of the nucleus and the critical energy barrier can be reduced by either increasing the free energy of the bulk crystal $(\Delta G v)$ or by reducing the surface free energy $(\gamma)$. An increase in the supersaturation can increase the $(\Delta G v)$ leading to an overall decrease in the critical energy barrier. Also, a decrease in the system temperature can lead to an overall decrease in $r^{*}$ and $\Delta G^{*}$.

\subsection{Rate of nucleation}

The rate of nucleation per unit volume can be defined as the number of nuclei that can form in a solution per unit time. It is considered as one of the most important factors that determine the properties of the nanoparticles (Dimo Kashchiev, 2000; Sinha et al., 2013; Vekilov, 2010). A rapid and uniform nucleation process forms smaller particles with a narrow size 
distribution (Sinha et al., 2013; Vekilov, 2010). This can be explained by the spontaneous formation of many crystals, which causes a significant and a rapid drop in the supersaturation level. As a result of the rapid reduction of the supersaturation level, the nucleation process is terminated and the growth process starts, where most of the particles grow to the same size. However, slow nucleation causes a slow drop in the supersaturation level, which allows the nucleation to occur over a longer period of time (Sinha et al., 2013; Vekilov, 2010). This can lead to a broad size distribution for the nanoparticles. Therefore the size of the nanoparticles can be controlled by controlling the rate of nucleation $(d N / d t)$ (Sinha et al., 2013). The Arrhenius equation (Eq. 8) describes all the factors that can have a significant influence on the rate of nucleation $(d N / d t)$. These are supersaturation $(S)$, surface free energy $(\gamma)$ and the temperature (T). $A$ is a pre-exponential factor (Thanh et al., 2014). A supersaturated system possesses a low critical energy barrier and has a high nucleation rate. Supersaturation was found to be the factor with the largest effect on the nucleation rate, as shown in Figure 2 (Thanh et al., 2014).

$$
\frac{d N}{d t}=A \exp \left(16 \pi \gamma^{3} v^{2} / 3 k^{3} T^{3}(\ln S)^{2}\right) \quad \text { Eq. } 8
$$
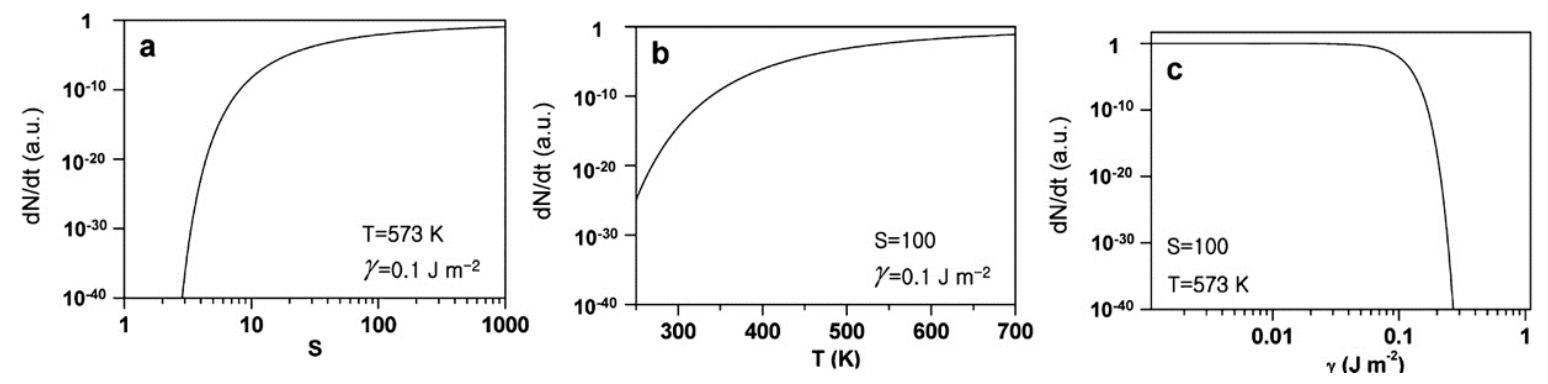

Figure 2. The effect of the a) supersaturation, b) temperature, and c) surface free energy on the nucleation rate (reprinted with permission from (Thanh et al., 2014)). 
In the antisolvent nanoprecipitation process, the mixing of the solvent and the antisolvent pose a significant effect on the uniformity and the rate of nucleation, which consequently control the size and surface properties of the nanoparticles (Sinha et al., 2013). An efficient and uniform mixing ensures a uniform supersaturation throughout the solution (Sinha et al., 2013). Consequently, this induces a uniform nucleation, which results in the formation of smaller particles with a narrower size distribution in comparison to non-uniform mixing (Figure 3) (Dimo Kashchiev, 2000; Sinha et al., 2013). These factors are discussed more in detail later in section 3 .

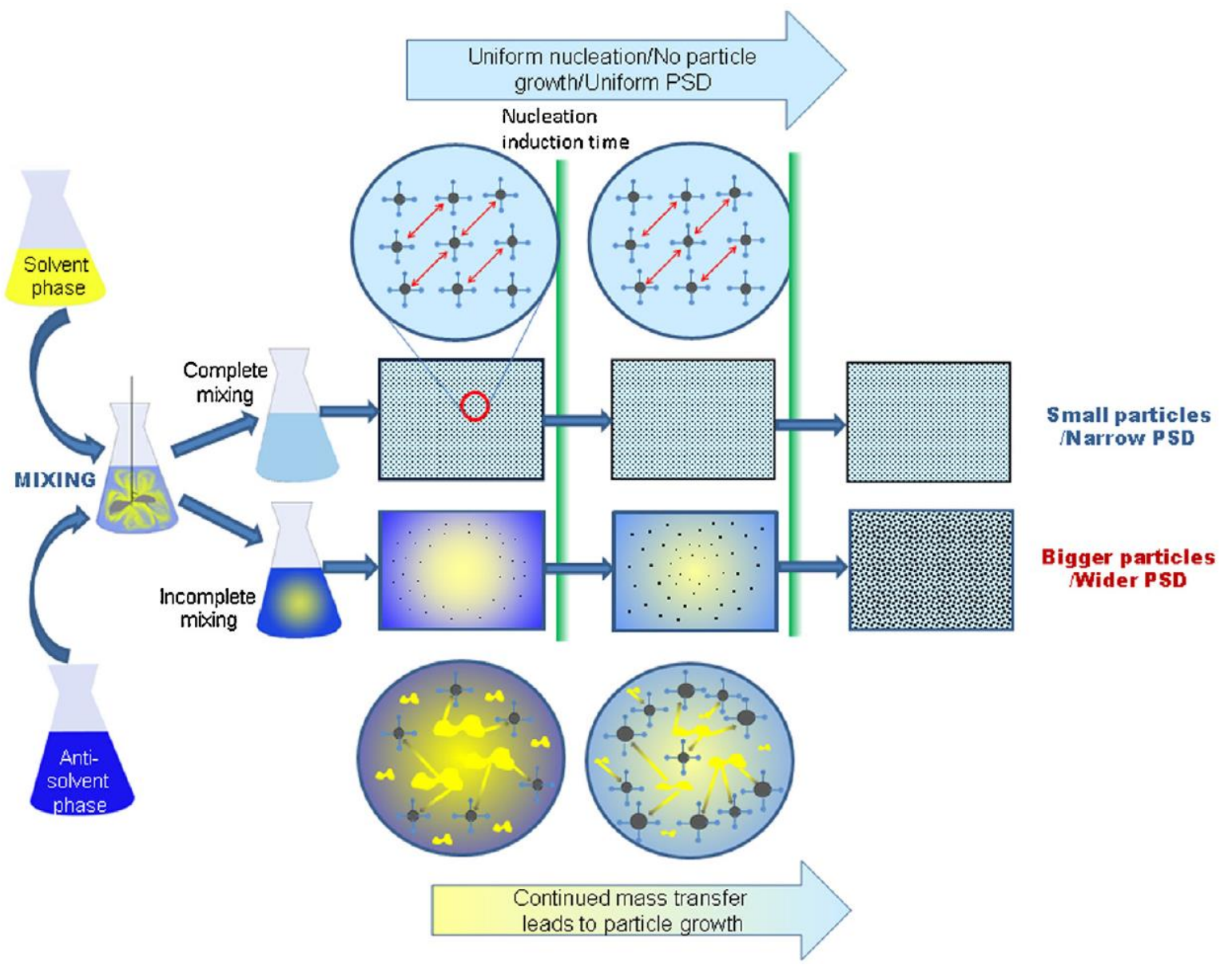

Figure 3. Illustration of the effect of the mixing efficiency on the final particle sizes (reprinted with permission from (Sinha et al., 2013)).

\subsection{Classical growth theory}


A rapid and uniform nucleation is a prerequisite for fabricating nanoparticles with small particle size and narrow size distribution (Sinha et al., 2013). However, the size distribution can be affected by both the nucleation process and the kinetics of the growth process (Cao and Wang, 2011; Sinha et al., 2013). Nucleation and growth occur simultaneously when the solute concentration exceeds $C_{\min }$ (Sinha et al., 2013). However, the rate at which each process proceeds can be different (Cao and Wang, 2011). In the case of homogenous nucleation, rapid nucleation ensures that all nuclei form simultaneously. These nuclei undergo a similar growth process over time, which lead to the production of monodisperse nanoparticles. However, this does not apply when the nucleation occurs over a long period of time (Cao and Wang, 2011).

The growth process starts with the formation of the reactants named as "growth species". The growth species diffuse and are adsorbed onto the surface of the nucleus, leading to a growth in size (Cao and Wang, 2011). The continuous growth of the particle size can be controlled by either diffusion or surface mechanisms (Cao and Wang, 2011). Each one of these processes can lead to particle population with a different size distribution (Cao and Wang, 2011).

\subsubsection{Diffusion-controlled growth}

The nucleation process stops when the solute concentration drops below $C_{\min }$, and the growth of the nanoparticles proceeds as illustrated in Figure 4. If the growth of the nanoparticles is controlled by the diffusion of the monomers from the bulk solution to the solid surface of the nuclei, the growth will result in the formation of monosized nanoparticles (Cao and Wang, 2011; Sinha et al., 2013). This can be described by Eq. 9, where $r$ is the radius of the particle, $D$ is the diffusion coefficient of the solute molecule, $C$ is the concentration of the monomers 
in the bulk, $C s$ is the concentration of the monomers at the solid liquid interface and $V m$ is the molar volume of the nucleus (Sinha et al., 2013). By solving the equation and assuming that $r_{o}$ is the initial radius of the nucleus, the equation can be rearranged to Eq. 10 . As $K_{D}=2 D(C$ Cs) Vm, the equation can be written as in Eq. 11 (Cao and Wang, 2011).

$$
\begin{array}{ll}
\frac{d r}{d t}=\frac{D(C-C s) V m}{r} & \text { Eq. } 9 \\
r^{2}=K_{D} t+r_{o}^{2} & \text { Eq. } 10
\end{array}
$$

Assuming that the initial radius difference for two particles is $\delta r_{o}$, the radius difference $(\delta r)$ decreases as time increases or the particles grow bigger as illustrated in Eq. 11 (Cao and Wang, 2011). By combining Eq. 10 and 11 into Eq. 12, it can be proved that the radius difference decreases over time. This explains how diffusion-controlled growth produces particles with a narrow size distribution (Cao and Wang, 2011; Sinha et al., 2013).

$$
\begin{array}{lr}
\delta r=r_{o} \delta r_{o} / r & \text { Eq. } 11 \\
\delta r=r_{o} \delta r_{o} /\left(K_{D} t+r_{o}^{2}\right)^{1 / 2} & \text { Eq. } 12
\end{array}
$$

There are some suggested practical methods to achieve growth driven by diffusion, which is desirable for making monodisperse nanoparticles. These include increasing the viscosity of the bulk solution or introducing a layer on the surface of the nanoparticles as a barrier for the monomer's diffusion (Cao and Wang, 2011). These methods ensure that the diffusion is the limiting step for the growth process. 




Figure 4. Illustration of the temperature and solute concentration effects on nucleation and growth processes (reprinted with permission from (Sinha et al., 2013)).

\subsubsection{Surface-controlled growth}

The growth is dominated by surface processes when the diffusion of the monomers from the bulk solution to the surface of the particles occurs rapidly (Cao and Wang, 2011). Two main mechanisms drive surface-controlled growth. These are mononuclear growth and polynuclear growth (Cao and Wang, 2011). In monolayer growth the particles have sufficient time to grow because growth occurs sequentially layer-by-layer. In this case the growth rate is proportional to a particle's surface area (Cao and Wang, 2011). This means that mononuclear growth is not suitable for making mono-sized nanoparticles, as is illustrated in Figure 5. However, in poly-nuclear growth the surface concentration of the reactant is very high, and the process occurs sufficiently rapidly for the second layer to begin forming before the first layer is completed. In this case the growth rate is relatively uniform and depends only on temperature. Therefore, poly-nuclear growth is considered to be favourable for making monodisperse nanoparticles (Cao and Wang, 2011; Sinha et al., 2013). 
Whether the growth process is diffusion-controlled or surface-controlled is indicated by the size of the Damköhler number ( $D a)$ (Sinha et al., 2013). This dimensionless number represents the ratio of $t_{\text {mixing }}$ to $t_{\text {precipitation }}$, where $t_{\text {mixing }}$ is the time required to achieve complete homogenous mixing and $t_{\text {precipitation }}$ is the time required to allow the drug concentration reaching the critical nucleation concentration $\left(C_{n}\right)$ and initiating the formation of the nuclei. If $D a$ is much less than 1, the reaction is controlled by diffusion (Sinha et al., 2013). If $D a$ is much larger than 1, the reaction is surface controlled. If $D a$ is on the order of 1 both surface processes and diffusion are important.

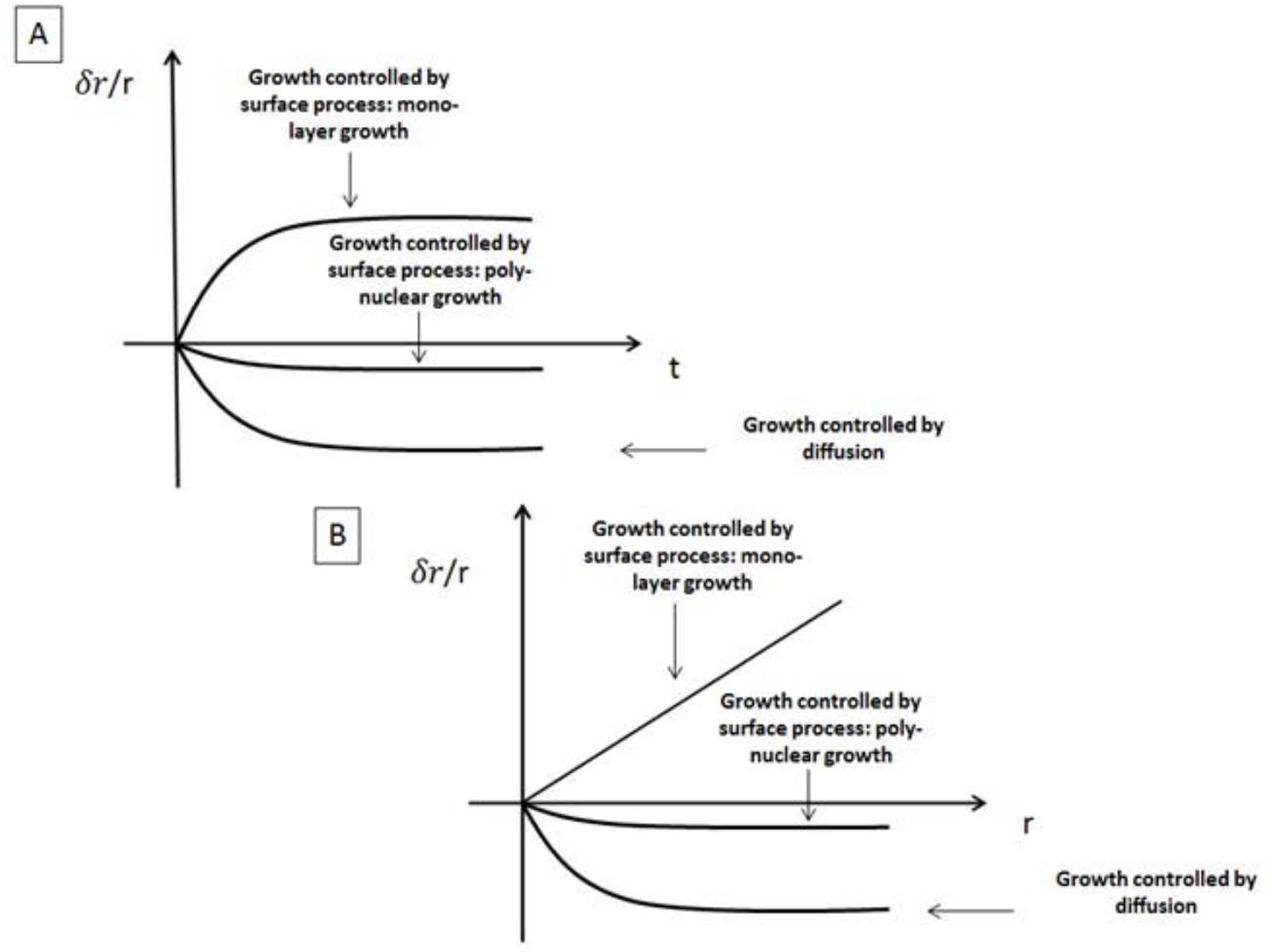

Figure 5. Schematic illustration of the changes of the radius difference between two particles $(\delta r)$ during the growth process as functions of (A) growth time and (B) particle size for all mechanisms of subsequent particle growth. (Reprinted with permission from (Cao and Wang, 2011)). 


\section{Experimental considerations}

\subsection{General factors affecting nanoprecipitation process}

The theoretical background described above forms the fundamental principle of nanoparticles formation by nanoprecipitation method. Experimentally, the precipitation process is regulated by several parameters and processes as illustrated by Figure 6. These variables are interdependent; therefore, it is difficult to discuss them completely in isolation.

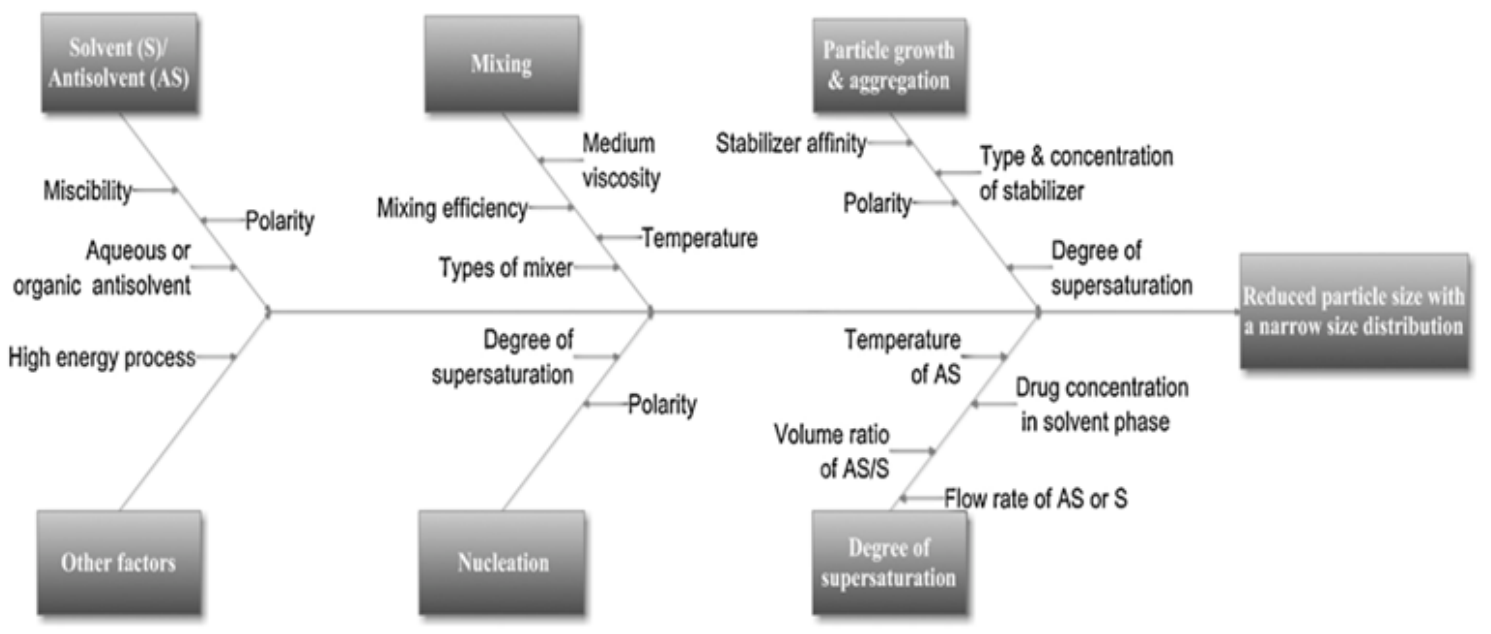

Figure 6. Fish-bone diagram depicting various interdependent factors influencing the quality attributes of precipitation process (reprinted with permission from (Sinha et al., 2013)).

\subsubsection{Degree of supersaturation}

Degree of supersaturation is the ratio between drug concentration in the binary mixture of solvent and aqueous-based antisolvent after mixing and its equilibrium solubility in that mixture. Supersaturation, in most cases, is the driving force of the precipitation process (Haberkorn et al., 2003). The degree of supersaturation is expressed by Eq. 13, in which $C$ is the drug concentration in the solvent and $C_{E q}$ is the saturated drug concentration under equilibrium in the binary mixture. In principle, increasing the degree of supersaturation increases the nucleation rate and subsequently decreases the size of the precipitated particles. 


$$
\text { Degree of supersaturation }=\frac{C}{C_{E q}}
$$

Eq. 13

As illustrated in Figure 7, in the case of low degree of supersaturation, the system will spend limited time in the supersaturation phase (where the nucleation happens homogenously spontaneously with minimum crystals growth) and much longer time passing through the metastable zone (where the crystals growth predominates over the nucleation) which ultimately will produce larger poly-dispersed particles in comparison to those produced at a higher degree of supersaturation. However, high degree of supersaturation shortens the

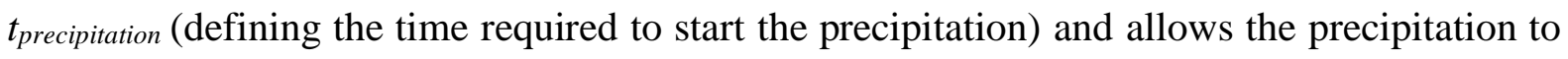
start before homogenous mixing can be reached $(D a<1)$. This results in the fabrication of large poly-dispersed particles unless a very efficient mixing tool/process is used (Tao et al., 2019).

Increasing the degree of supersaturation of either a drug or a polymer could be achieved by manipulating the antisolvent phase (increasing the volume or decreasing mixing temperature) or the solvent phase (increasing the volume or drug/polymer concentration). Such manipulation may also have a subordinate effect on the precipitation process. For instance, increasing the volume of the antisolvent decreases the overall drug/polymer concentration, which lowers the collision rate and reduces the probability of nanoparticles aggregation. On the contrary, increasing the volume of solvent phase boosts the Ostwald ripening effect due to the influence of the dissolved drug molecules in the residual solvent (Kakran et al., 2012). 


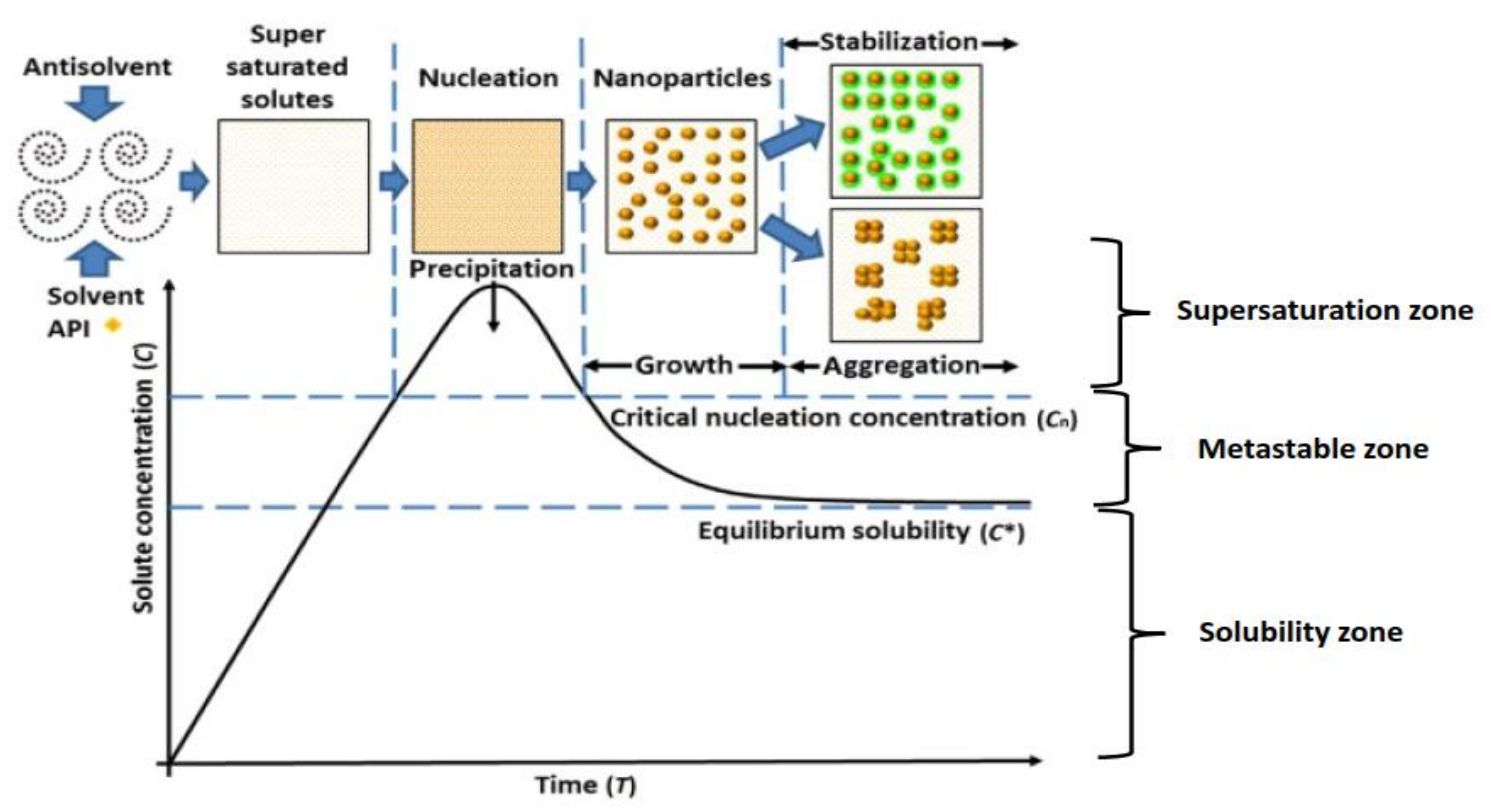

Figure 7. The effect of the degree of supersaturation on the process of precipitation (reprinted with permission from (Tao et al., 2019)).

\subsubsection{The mixing process of solvent and antisolvent}

The mixing process of the solvent and the antisolvent is considered to be the most crucial step for producing nanoparticles with small particle size and narrow size distribution. Generally, a uniform mixing is a prerequisite to achieve a uniform supersaturation throughout the bulk solution, which triggers a uniform nucleation. The Ostwald ripening effect is neglected when monodisperse nanoparticles are formed, which enhances the physical stability of the resultant particles (Thorat and Dalvi, 2012) (Figure 7).

The uniformity of the mixing of the solvent and antisolvent is heavily dependent on the mode of flow when the mixing proceeds. In turbulent flow, which occurs in conventional mixing methods such as magnetic stirrers, the mixing process can be subdivided into three mixing scales: macro-, meso-, and micromixing. Macro-mixing encompasses the macroscopic flow processes on the decimetre scale, while meso-mixing describes mixing processes on the 
millimetre scale, which are based on turbulent vortex breakdown. Micro-mixing represents the mixing on the smallest scales of motion (known as Kolmogrov scale) which in most cases relies on molecular diffusion as a driving force for mass transfer (Mohammad et al., 2008).

Once the mixing is completed, the whole precipitation process could be chronologically divided into three distinct time scales; the first is the precipitation time, $t_{\text {precipitation }}$, also known as the nucleation induction time. At $t_{\text {precipitation }}$ the drug concentration reaches the critical nucleation concentration $\left(C_{n}\right)$, which initiates the formation of the nuclei. The second phase is the growth time, $t_{G}$, during which the precipitated nuclei start to grow by further accumulation of dissolved drug/polymer molecules on the newly precipitated nuclei, as detailed in section 2.3. Finally, during the aggregation phase, the grown, precipitated nuclei collide and aggregate (Martínez Rivas et al., 2017) (Figure 7).

The homogeneity of the precipitation process is indicated by the size of $D a$. Ideally $D a$ should be below 1 in order to achieve a homogenous precipitation and produce small and uniform nanoparticles. This ensures a complete uniform distribution of the solvent phase within the antisolvent phase before the precipitation step starts. This could be achieved by either lowering $t_{\text {mixing }}$ (through enhancing the mixing efficiency) or increasing $t_{\text {precipitation }}$ (through increasing the viscosity to reduce molecular mobility or using stabilizers) (Chan and Kwok, 2011).

Multitudinous methods/tools with different mixing efficiencies have been assessed to be used to perform the mixing step. These include simple stirring using magnetic stirrers (Freag et al., 2013; Shariare et al., 2019), static mixers (Dong et al., 2010) (in which solvent and antisolvent fluids are pumped at high rates (litres per min) through a small nozzles to hit 
mixing elements (such as blades) to induce turbulent mixing), and the recently introduced techniques that uses microfluidics including micromixer chips, and high energy input jet stream mixers (microfluidizers) (Salazar et al., 2014). Recently, the use of microfluidic systems of different channel and mixing junction geometries has been introduced to provide a controlled, tuneable and continuous scalable mean for efficient fluid mixing and nanoparticles production. This is further discussed in section 3.2.

Simple mixing, which is performed by conventional mixing with active stirrers, is often not sufficient to produce small monodisperse drug/polymer nanoparticles in moderate concentrations of the drug/polymer. Thus high energy processes are required to aid in the development of small monodisperse nanoparticles (Freag et al., 2013; Zhang et al., 2006). Examples of such high energy processes include ultrasonication (Dhumal et al., 2008; Gajera et al., 2019), high pressure homogenization (Salazar et al., 2014), and high shear homogenization (Karakucuk et al., 2019; Yu et al., 2018). The small particle size obtained by these methods is often a result of the improved mixing efficiency due to cavitation (in the case of ultrasonication) and turbulent currents which minimise particle growth after nanoparticles precipitation (Sinha et al., 2013). During these high-energy processes, the drug/polymer is firstly precipitated after the addition of the antisolvent phase.

In the case of drug molecules being the solute, unstable fragile form of nanoparticles is firstly obtained in the initial stage of precipitation, which may be amorphous, semi-crystalline or super-cooled liquid form. This unstable form is then transformed to a more stable crystalline drug particle in the presence of high energy during annealing. Throughout the annealing process, the highly energetic thermodynamically unstable form of the precipitated particles is converted into a form of lower energy and higher stability via single or repeated application 
of energy (from mechanical stress or the generated heat), followed by thermal relaxation (Sinha et al., 2013).

\subsubsection{Solute (drug /polymer) concentration in solvent phase}

Solute (which could be either drug or polymer in the cases of making drug or polymeric nanoparticles) concentration in solvent phase is another key factor to manipulate the degree of supersaturation. As illustrated in Figure 4, Increasing solute concentration in solvent phase will increase the total content of the solute in the binary mixture of the solvent and antisolvent, thus increases the degree of supersaturation causing rapid and uniform nucleation and producing small nanoparticles (Dalvi and Dave, 2009). However, when the solute concentration is too high (above the optimum solute concentration in Figure 8), there is a potential risk for increasing the number of nuclei and their collisions that promote particle growth and aggregation. Therefore, the benefit of increasing solute concentration for producing small and uniform nanoparticles is concentration dependent (Gajera et al., 2019), as demonstrated in Figure 8. This was evaluated by many studies that reported a direct increase in the particles size with increasing the polymer concentration (Chorny et al., 2002; Nehilla et al., 2008; Seremeta et al., 2013). In addition, the drug solubility in the solvent plays a crucial role in determining the degree of supersaturation and the volume of solvent required. It, in turn, affects the stability of the formed nanoparticles due to the Ostwald ripening effect of the residual solvent in case of using a large volume of the solvent (Gajera et al., 2019). 


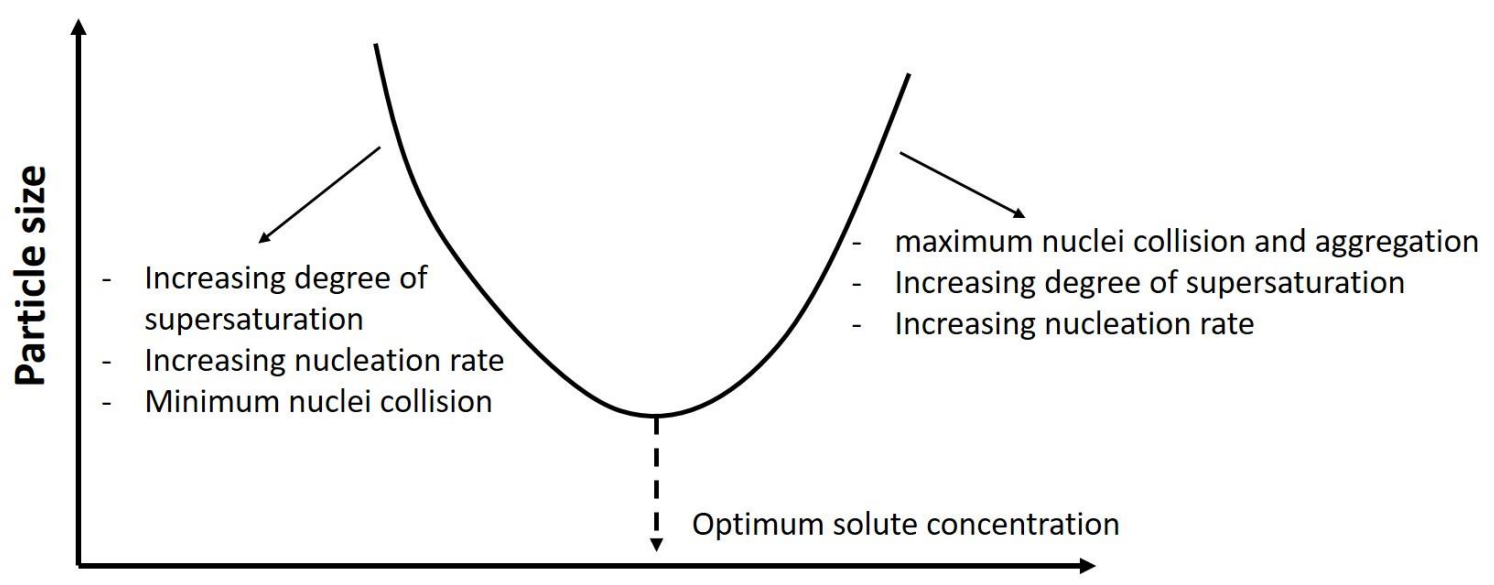

Solute concentration in solvent phase

Figure 8 . The effect of solute concentration in solvent phase on the particle size of the precipitated particles.

\subsubsection{The volume ratio between the solvent and the antisolvent (S-AS ratio)}

The degree of supersaturation of the solute (drug/ polymer) can also be manipulated by varying the solvent/antisolvent volume ratio (S-AS ratio). As shown in Eq. 13, with keeping the same solute (drug/polymer) content, increasing S-AS ratio will decrease the degree of supersaturation through increasing the solute equilibrium saturated solubility in the binary mixture (Kakran et al., 2012; Wang et al., 2007). Furthermore, the high solvent content will induce particle growth due to Ostwald ripening after particle precipitation (Gajera et al., 2019; Verma et al., 2011). Moreover, selecting a solvent that has a good solute dissolving capacity is prominent to minimize its volume and ease its removal after the precipitation step has been achieved. Different findings have been reported by several studies. For example, Budhian et al. reported a decrease in particle size with an increase in drug loading efficiency when the solvent volume is decreased (Budhian et al., 2007). This was attributed to the short solvent evaporation time, which allows the particles to transfer from the liquid state to the locked state rapidly. However, a contradictory finding was reported by Limayem Blouza et 
al.. They found that the S-AS volume ratio was decreased by increasing the aqueous phase volume (Limayem Blouza et al., 2006; Nagy et al., 2019). The size of the nanoparticles was found to increase from $320 \pm 12 \mathrm{~nm}$ to $536 \pm 14 \mathrm{~nm}$, with a drop in the drug encapsulation efficiency from $96.21 \%$ to $75.93 \%$, by increasing the water (as the antisolvent) volume from $50 \mathrm{ml}$ to $100 \mathrm{ml}$ respectively (Limayem Blouza et al., 2006). This suggests that the properties of the nanoparticles can be tuned not only by varying the A-AS ratio but also through the means by how it is changed.

\subsubsection{Type and concentration of the stabilizer}

The choice of the type and the concentration of the used stabilizer are crucial to assure good stabilization of the nanoparticle surfaces against growing and aggregation. A wide variety of stabilizers have been used, some being polymeric (non-ionic, linear, hydrophobic or amphiphilic), while others being surfactants (ionic or non-ionic) (Van Eerdenbrugh et al., 2009). The selection of an effective stabilizer should be based on its affinity to be adsorbed on the particles' surfaces. The higher the affinity of stabilizer to drug/polymer, the faster it adsorbs and occupies the particle surface; hence smaller particles can be produced. The adsorption of the stabilizers (that have surface activity) can decrease the surface free energy of the new-born nuclei and lower the tendency of these nuclei to grow or aggregate. In addition, some stabilizers stabilize the formed particles either sterically (due to long polymeric chains) or statically (due to its charge) to prevent their aggregation during storage. In some cases, blends of both steric and static stabilizers are used to provide the most optimal stabilization effect (Verma et al., 2011).

The appropriate stabilizer concentration is considered as another vital parameter that is interdependent on the nature of the stabilizers and the precipitated solute. Surfactants and 
polymeric stabilizers possess different mechanisms for nanoparticles stabilization. Increasing the surfactants concentration increases the adsorbed fraction of molecules onto the particles' surfaces. This improves the colloidal stability of the nanoparticles. However, there is a concentration limit. If the surfactants concentration exceeded the (critical micellar concentration), an Ostwald ripening effect can be promoted due to micelles formation. Similarly, If the used polymeric stabilizer concentration exceeds the critical flocculation concentration (CFC), the polymeric chains start to flocculate and get entangled together which promote aggregation and size enlargement (Thorat and Dalvi, 2012). The influence of the used stabilizer and its concentration have been widely investigated, yet seemed contradictory results were reported. This emphasizes the effect of the stabilizer being highly system dependent and even slight difference in material and processing parameters could lead to the deviation of results. For example, while Dong and Feng (2004) reported no significant change in the MPEG-PLA nanoparticle size when Pluronic F68 concentration was increased (Dong and Feng, 2004), Zweers et al. reported a significant increase in particle size when high PVA concentrations were used (Zweers et al., 2003), and Allemann et al. reported a continuous decrease in particle size with increasing PVA concentrations (Allémann et al., 1992). These seemingly contradictory results were systemically analysed by other research groups (Lamprecht and Arica, 2005; Nagy et al., 2019). They proposed that the size of the resultant nanoparticles could be affected by two competing effects. These are the enhanced interfacial stability achieved by the higher PVA concentration (which promotes size reduction) and the increased viscosity of the aqueous phase (which reduces the mixing efficiency leading to a larger size population of nanoparticles). The dominant factor is determined by the system and the processing parameters (Nagy et al., 2019), which reinforces the need to understand the effect of the processing parameters for each nanoprecipitation system. 


\subsubsection{Solvent and antisolvent miscibility}

Most of the precipitation processes use an organismic solvent phase to mix with an aqueousbased antisolvent phase. The miscibility of the solvent with the aqueous-based antisolvent is one of the critical factors when choosing the solvent systems, as it is expected to affect the mixing step and the solute diffusion into the antisolvent phase. Consequently, this affects the homogeneity of the nucleation step and the mean particle size of the precipitated nanoparticles. Cheng and co-workers studied the effect of using a range of solvents with different water miscibility on the particle size of the produced PLGA NPs (Cheng et al., 2007). It was found that the solvents with high miscibility with water (e.g. DMF, dimethyl formamide) led to the production of smaller NPs in comparison to that produced by solvents of lower water miscibility (e.g. THF, Tetrahydrofuran). However, Guhagarkar and coworkers reported a significant decrease in nanoparticles size when a mixture of THF and acetone was used as the solvent phase, explained by the rapid diffusion of the acetone into the aqueous antisolvent phase favouring the formation of smaller nanoparticles (Guhagarkar et al., 2009; Nagy et al., 2019).

\subsubsection{Other factors}

Some other factors impose a contributing effect in controlling the particles size distribution and the physicochemical properties of the produced nanoparticles. For instance, reducing the temperature of antisolvent was reported to decrease the particle size and narrow the particle size distribution (Xia et al., 2010). That could be attributed to the reduction in the equilibrium solubility of the solute in the binary mixture that in turn increases the degree of supersaturation. Moreover, lowering the temperature increases the system viscosity and decreases the particles movement, which decreases the particles growth and collision rates 
giving more time to the stabilizers to anchor on the particles' surfaces and preventing their aggregation (Dalvi and Dave, 2009; Zhang et al., 2009). It also has been reported that the nature of both solvent and antisolvent is able to affect the crystallinity and polymorphic form of the produced nanoparticles (Zhang et al., 2006).

\subsection{Microfluidic systems as a tuneable tool for efficient mixing}

Recently, the use of microfluidic systems has been increasingly discussed as a means of efficient tuneable mixing which is vitally important for producing small nanoparticles with narrow size distribution. The tuneability of the particle size can be well controlled by systemic screening of the parameters used for the micro-mixing process used in microfluidic methods (Dietzel, 2016; Feng et al., 2016; Hessel et al., 2005; Riahi et al., 2015; Sanjay et al., 2018). The micro-mixing process can be categorized as either active or passive mixing. Active mixing in a microchip is tightly controlled by the external energy input, or by ultrasonication (Yang et al., 2001) or via an electrokinetic instability (Oddy et al., 2001). In passive mixing, the geometry of the microchip is designed to promote mixing within the flow without external influence (Hessel et al., 2005; Suh and Kang, 2010). Since passive mixing depends only on the microchannel geometry and the imposed flow conditions at the inlet, it is readily amendable to downscaling. Furthermore it is the most commonly discussed technique for the microfluidic manufacture of nanoparticles. For these reasons we focus the present discussion on passive mixing.

Passive mixing is strongly influenced by the prevailing flow within the microchannel. The flow is strongly affected by the viscosity and density of the fluid, by the geometry of the microchip, and by the surface properties of the channel walls. Typical flow regimes are 
characterised by the dimensionless Reynolds number, which expresses the ratio between fluid inertia and the viscous shear force and is defined as

$$
\text { Re }=\frac{\text { Inertial forces }}{\text { Viscous forces }}=\frac{\rho V d}{\mu} \quad \text { Eq. } 14
$$

Here $V$ is a typical speed that is representative of the flow, $d$ is a characteristic length (for example this might be the channel diameter in the case of a linear bath configuration), and $\rho$ and $\mu$ are the density and dynamic viscosity of the fluid respectively. Roughly speaking the flow is laminar if $R e$ is small and turbulent if $R e$ is large (Bally et al., 2012; Liu et al., 2015; Marre and Jensen, 2010). Since the channel diameter $d$ is on the order of micrometres, the Reynolds number is typically small and laminar flow is expected (Dietzel, 2016; Martins et al., 2018; Van Der Woerd et al., 2003; Weigl et al., 2003). Laminar flow is smooth with wellordered streamlines; in contrast turbulent flow is highly irregular and is characterised by small-scale flow structures and by rapid and chaotic fluctuations. Microfluidic systems often operate at Reynolds numbers below 1, wherein viscous forces dominate over inertial effects in a regime known as creeping flow or Stokes flow. However, specialized high-pressure microfluidic systems can also provide turbulent mixing conditions: examples include confined impinging jets mixers (CIJM), multi-inlet vortex mixers (MIVM) (Richter et al., 2013) and high pressure homogenization devices (microfluidizers) made from steel (Ebert et al., 2017).

Mass transfer is crucial for mixing within microchannels. Mass transfer occurs either by molecular diffusion, whereby molecules migrate across the interface separating the different fluid phases to be mixed, or by advection, whereby mass is carried directly by the prevailing fluid flow. The relative contribution to the mass transport of both of these mechanisms can be quantified by the Peclet number, $P e$, which is defined as (Eq. 15)

$$
P e=\frac{\text { advective transfer rate }}{\text { diffusive transfer rate }}=\frac{V d}{D} \quad \text { Eq. } 15
$$


Given the small channel diameters involved in microfluidic systems ( $d$ is measured in micrometres), $P e$ is typically small and diffusion provides the principal mechanism for mass transfer (Enrica Chiesa et al., 2018).

\subsubsection{Laminar flow microchips}

Laminar flow microchips (low $R e$ ) represent the majority of reported microfluidic systems. Typically, they are made of polydimethylsiloxane (PDMS) and/or glass and they contain channels with typical diameters in the micrometre range (e.g. $100 \mu \mathrm{m}$ to $500 \mu \mathrm{m}$ ) (Figure 9). Typical flow rates in these systems are moderate and do not exceed $3 \mathrm{ml} / \mathrm{min}$ (high flow rates would generate large pressures and cause PDMS chips to detach from the supporting glass slide). Usually the Peclet number $P e$ is less than 1 so that molecular diffusion provides the main means of the mass transfer in the microchip. This type of microfluidic systems (glass or PDMS microchips) has been widely used to produce both drug nanosuspensions (Amani et al., 2014; Panagiotou et al., 2009; Rahimi et al., 2017; Schianti et al., 2011) and polymeric nanoparticles (Donno et al., 2017; Karnik et al., 2008).



Figure 9. Laminar flow microchips made of (a) glass and (b) PDMS. (reprinted with permission from (Kimura et al., 2016) and (Feng et al., 2015), respectively)

\subsubsection{Turbulent flow high-pressure devices for nanoparticle generation}


These devices are made mainly from metal (aluminium or steel) to withstand high-pressure accompanying high flow rates (commonly range from 40 to $120 \mathrm{ml} / \mathrm{min}$ ) of the fluids. These systems such as confined impinging jets mixer (CIJM), and multi-inlet vortex mixer (MIVM) (shown in Figure 10) use the same concepts of the precipitation process, but often in this context are referred as "flash nanoprecipitation" (Saad and Prud'Homme, 2016; Tao et al., 2019). These devices depend on pumping the two liquids at high speeds to hit each other at certain angles which induce the turbulent mixing. The mass transport in such devices is dominated by advective processes.

(a)



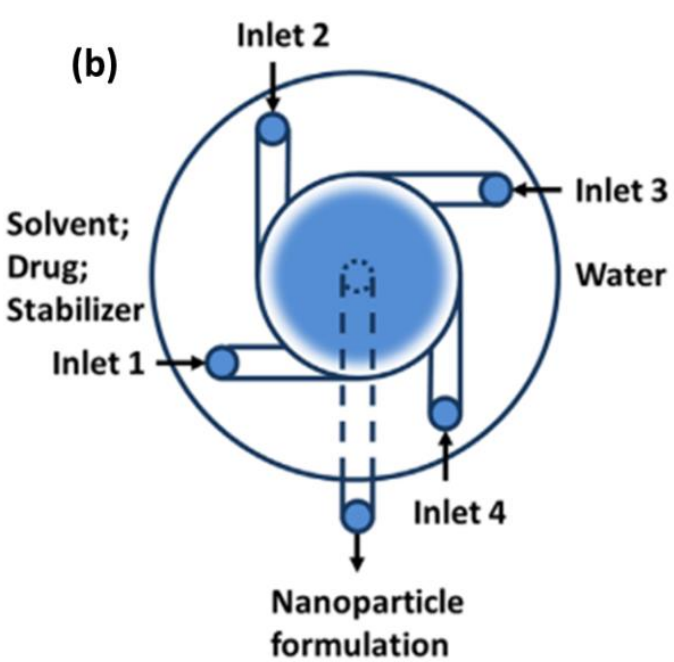

Figure 10. Schematic illustrations of (a) confined imping jet mixer (CIJM) and (b) multi-inlet vortex mixer (MIVM) (reprinted with permission from (Tao et al., 2019)).

\subsubsection{Accelerating mixing in microfluidic laminar flow microchips}

As was noted above, the flow in laminar flow microchips is smooth and regular and mixing cannot rely on turbulent flow processes. Given the small Peclet numbers involved, mass transfer tends to be dominated by diffusion. However, molecular diffusion is very slow: diffusive transport over a distance $L$ occurs over a timescale on the order of $L^{2} / D$, where $D i$ is the diffusivity of the substance being transported. Since $D$ is typically very small, the 
diffusion timescale over reasonable distances (here on the order of $100 \mu \mathrm{m}$ ) may be significant. Therefore the main objective when designing a micromixer is to limit the distance over which the solvent must diffuse. A general principle is to enlarge the contact area between the solvent and the antisolvent by stretching, splitting, folding or breaking up the solutions using disturbed flow that is induced by suitable microstructures within the channels. This creates thinner fluid layers and hence shorter distances for the solvent to diffuse across into the anti-solvent. Consequently this accelerates the mixing process and creates a preferential environment for a homogeneous precipitation of nanoparticles. In this context different types of mixers have been reported to be successful for producing nanoparticles (Dietzel, 2016).

- Lamination: parallel or sequential

- Segmentation: sequential or parallel

- Chaotic Advection

- Multi-phase flow

Parallel lamination mixers divide the different solutions to be mixed into a number $(n)$ of substreams which are then merged co-currently (Figure 11a). This requires minimal diffusion distances between the solutions and shortens the mixing time by a factor of $n^{2}$ in comparison to performing the passive mixing with a single channel. Sequential lamination mixers are made of a sequence of repeating transformation structures in each of which the solutions are divided into two sub-streams before being subsequently recombined (Figure 11b). Each split-and-recombine arrangement produces a doubling of the number of sub-streams. A fast reduction of the diffusion distance is achieved and with $n$ repetitions the mixing time reduces by a factor of $4^{(\mathrm{n}-1)}$. 

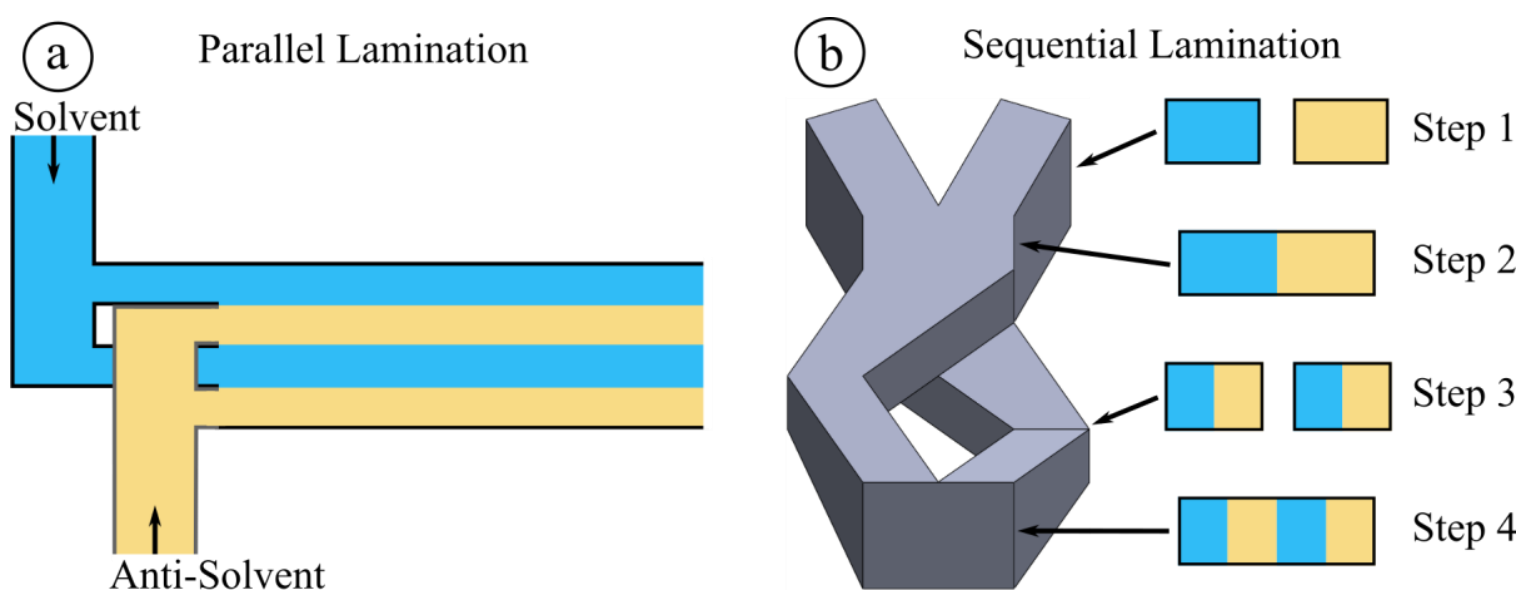

Figure 11. a) Schematic diagram illustrating parallel lamination of two fluids (solvent and anti-solvent) for passive mixing in microchannels. The grey lines represent sidewalls of microchannels, which are at a different height than the other; b) Schematic illustrating sequential lamination that shows a split and rejoin of the solvent and anti-solvent flowing through the microchannel. Several of these elements can be arranged in series to potentiate the effect. Each step of split and rejoin is also illustrated as cross-section view.

In sequential segmentation solvent and anti-solvent are injected into the main channel in an alternating periodic sequence (Figure 12a). In this process, the solutions are mixed via axial dispersion which can accelerate mixing by orders of magnitude (Nguyen, 2011; Nguyen and Huang, 2006). The size of the segments is important for determining the speed of mixing, which is also influenced by a periodic pressure pulsation at the inlet. Parallel segmentation requires an array of nozzles, which inject the solvent into the main channel carrying the antisolvent. The size of the individual nozzles and the velocity ratio of the inflowing solvent to the anti-solvent determine the surface area of the injected micro streams. Another approach is given by the integration of repetitive geometric changes or structural elements in the channel which leads to so-called chaotic advection (Figure 12b). It redirects the laminar flow into transversal paths by shear fields. The changes in direction of the flow lead to a permanent 
stretching, folding and twisting of the individual liquid layers during their passage through the channel. The strength of the shear forces on the flow is strongly dependent on the Reynolds number and on the structural elements within the channel. Such micromixers work efficiently only in a certain operational window: the shear forces can be too low if the Reynolds numbers are low, but if the Reynolds numbers are too high the structures lose their effect or unwanted secondary flows can occur. Micromixers with chaotic advection can therefore be classified according to three different flow ranges in which they perform efficiently: small $(1<\operatorname{Re}<10)$, medium $(10<\operatorname{Re}<100)$ and high $(100<\operatorname{Re})$ (Nguyen, 2011).
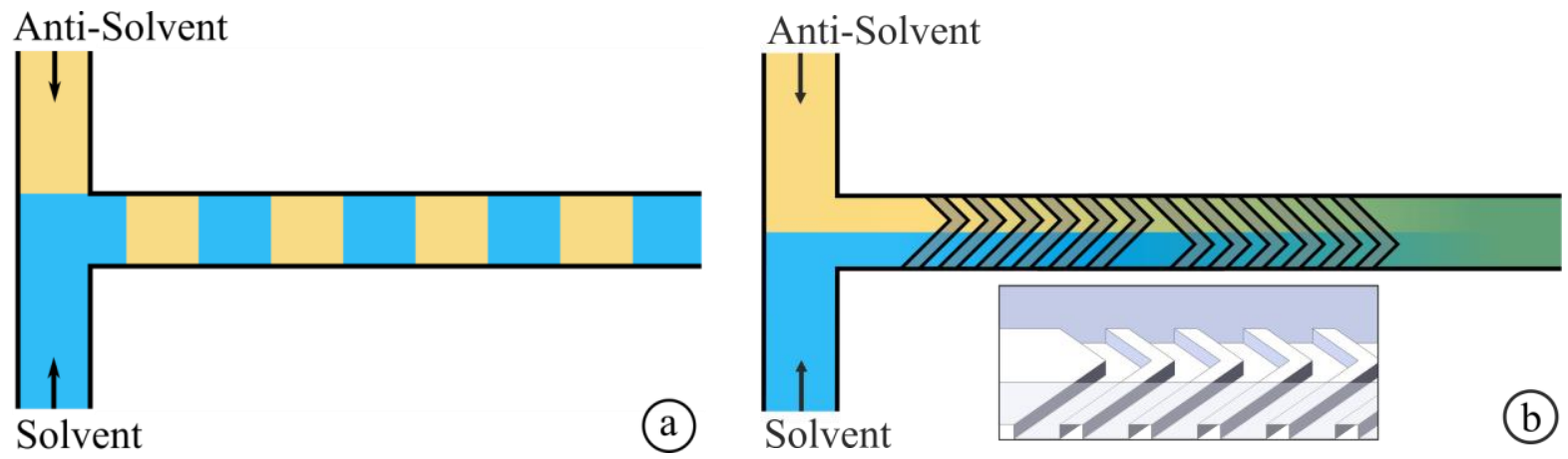

Figure 12. a) Schematic illustrating sequential segmentation. Segmentation is realized by alternately switching the volume flow of the individual solutions on and off; b) Schematic of a staggered herringbone micromixer for chaotic advection at low Reynolds number. Staggered herringbone grooves on the bottom of the main channel are arranged in repeating sequences each containing several grooves. Perspective view on channel structures in the herringbone micromixer (inserted picture).

A further possibility for increasing the efficiency of mixing is the use of multiphase flows, meaning that immiscible phases are admitted into a microchannel. Thereby small segments (plugs or droplets) are formed separated from each other by a more or less inert gaseous or 
liquid phase (Figure 13a). Within these segments the contact surface between the solvent and anti-solvent can be increased by internal vortices. Such multiphase flow is also known as Taylor flow (Gupta et al., 2010). To establish Taylor flow mixing the solvent and the antisolvent are combined at an intersection point and the flow is subsequently segmented with a gas phase into individual plugs. Shear forces on the channel walls create relative backflows in a segmented liquid plug, which induce vortices symmetrical to the channel central axes. The mixing time can be controlled by the size of the segmented plugs, which determines the size of the precipitated nanoparticles (Erfle et al., 2019).

A dramatic reduction of diffusion distances can also be achieved if droplets are formed with extremely small volumes on the order of nano- or femtoliters (Figure 13b). In some cases, to create such small droplets, the solvent and the antisolvent in a droplet can be transferred into a non-miscible medium (oil, for example) by flow focusing. The solutions within the droplet are subsequently mixed by diffusion (Günther and Jensen, 2006). As an alternative, the solvent can be injected as a droplet into the anti-solvent itself, which allows the solvent to diffuse from the droplet into the surrounding medium (Lorenz et al., 2018). The size of the nanoparticle is thereby determined by the amount of the dissolved active ingredient contained in a single droplet. 


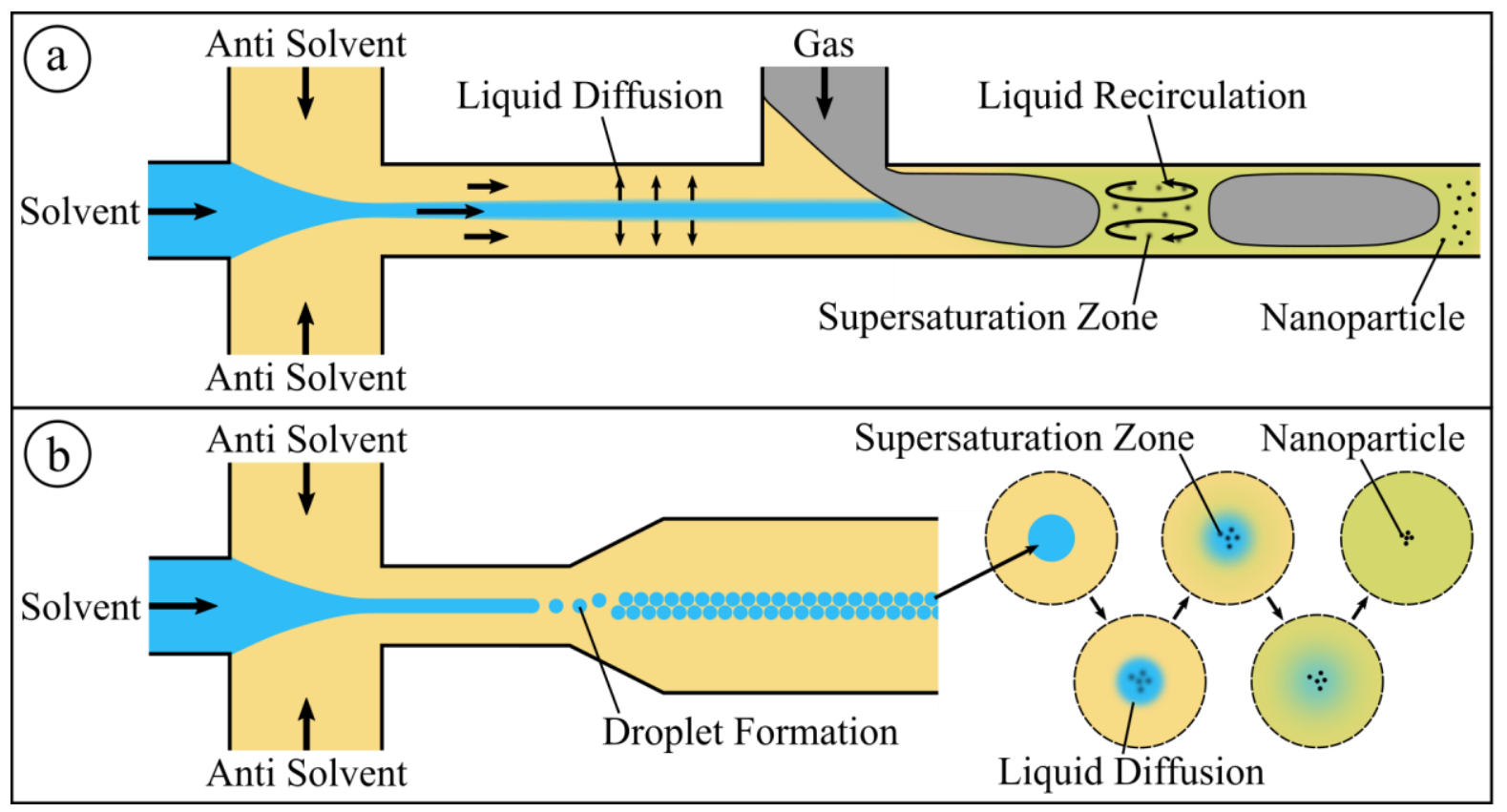

Figure 13. Schematic illustrations of two concepts of multiphase micromixers. A) Gas-liquid mixer (Taylor flow system), in which the solvent and anti-solvent are combined by flowfocusing and an injected gas phase segments the flow; b) Droplet formation, in which the solvent stream is narrowed with the antisolvent sheath flow leading to flow focusing. The filament stream breaks off into individual droplets. A suitable choice of solvent and a stabilizer is a necessary condition for a stable droplet formation

\subsubsection{Flow rate ratio (FRR)}

Flow rate ratio (FRR) is the ratio of the drug/polymer solution (solvent-S) flow rate to the antisolvent (AS) flow rate. As was discussed previously in section 3.1, changing the flow rate ratio (S/AS) simultaneously changes more than one factor including the degree of supersaturation (shown in Figure 14), the total drug concentration that affects the collision rate and particle aggregation, the solvent content that affects the stability of the produced nanoparticles, and the efficiency of the mixing process by modulating the path-length for diffusion (Figure 15). 


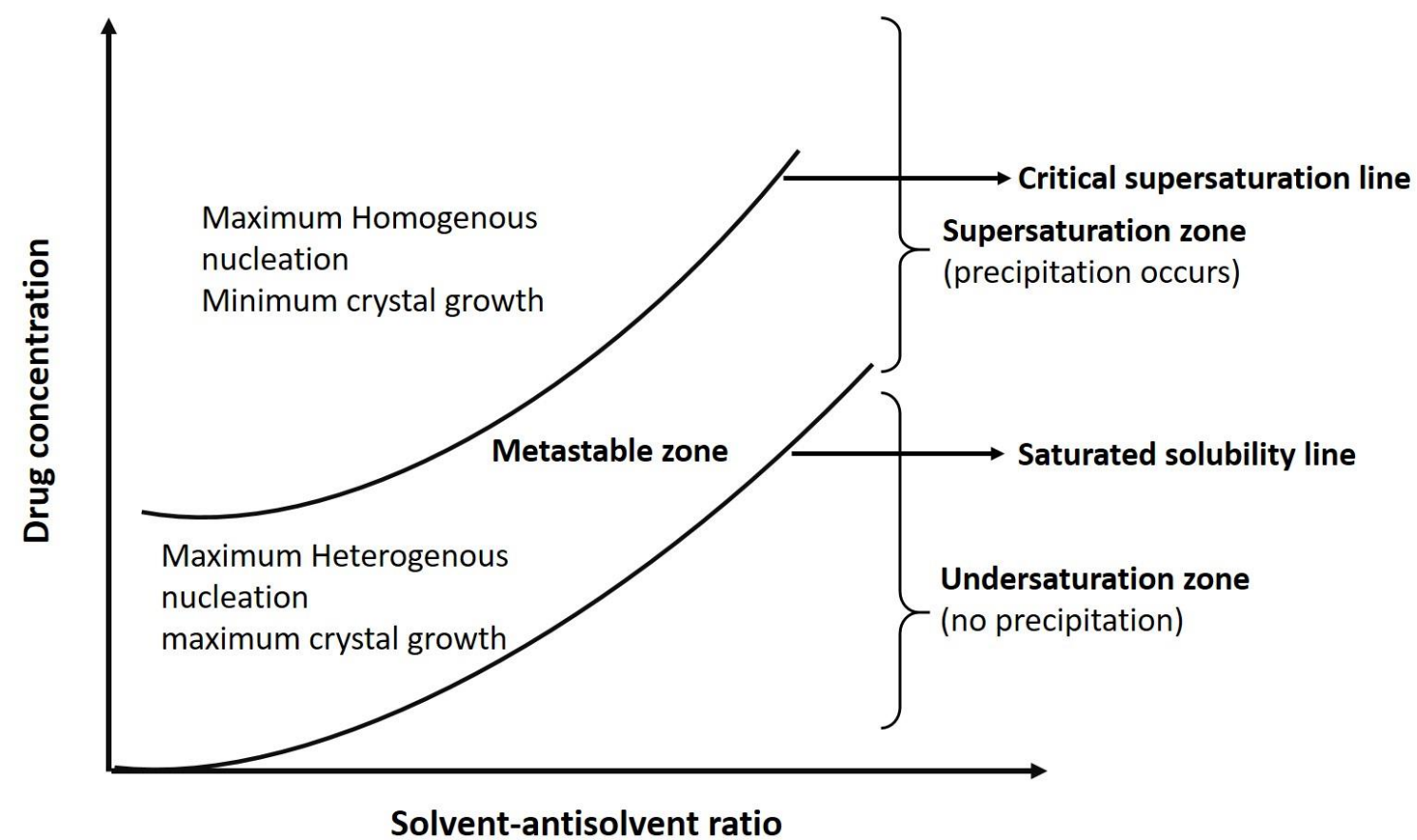

Figure 14. Schematic representation of the effect of the solvent anti-solvent ratio on the precipitation process (reprinted and modified with permission from (Nagy et al., 2019)).

As illustrated in Figure 14, increasing the S-AS ratio while maintaining the same drug content in the binary mixture will induce a relatively low degree of supersaturation, and hence a low nucleation rate with a higher crystal growth rate and, ultimately, larger particles. At a low S-AS ratio, a high degree of supersaturation (where homogenous nucleation dominates over crystal growth) could be easily attained even with low solute concentrations (Ali et al., 2009; Kulkarni and Myerson, 2017; Su et al., 2007; Zhao et al., 2007).

In the case of a flow focusing microchip that does not involve droplet formation (as shown in Figure 15), decreasing FRR makes the solvent stream sheathed by the surrounding antisolvent stream and decreases the path-length required for the drug molecule to diffuse from solvent to antisolvent stream which in turn reduces $t_{m i x}$ as shown in Eq. 16. 


$$
t_{m i x}=\frac{W_{f}^{2}}{4 D}=\frac{W^{2}}{9 D} \frac{1}{\left(1+\frac{1}{R}\right)^{2}}
$$

where $D$ is the diffusivity of the solvent, $W_{f}$ is the width of the internal focused stream, $W$ is the channel width, and $R$ is the flow rate ratio. To the best of our knowledge, these factors have not been investigated independently in any reported literature.

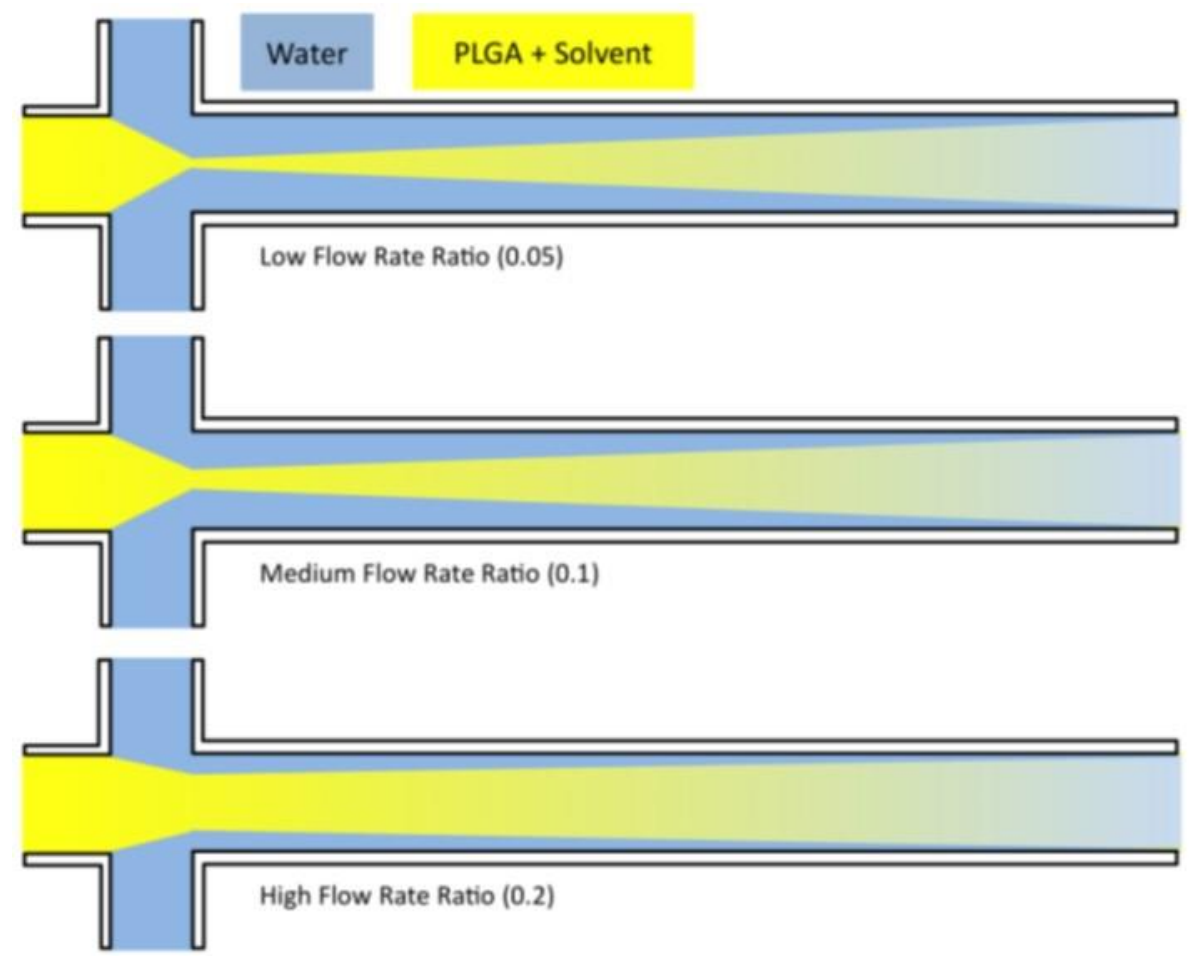

Figure 15. The effect of different FRR on the pathlength for diffusion within a flow focusing microchip. (reprinted with permission from (Lababidi et al., 2019)).

Baby T. et al (Baby et al., 2017) have investigated the effect of using different FRR on the particle size of PLGA nanoparticles using PLGA and PEG with different molecular weights (namely, PLGA $20 \mathrm{~K}-\mathrm{PEG}_{5 \mathrm{~K}}$ and $\mathrm{PLGA}_{55 \mathrm{~K}}-\mathrm{PEG}_{5 \mathrm{~K}}$ ). They found that increasing FRR from 0.1 to 0.8 increased the particle size from $32.0 \pm 0.6 \mathrm{~nm}$ to $62.6 \pm 0.4 \mathrm{~nm}$ for $\mathrm{PLGA}_{20 \mathrm{~K}}-\mathrm{PEG}_{5 \mathrm{~K}}$. This effect was more pronounced for $\mathrm{PLGA}_{55 \mathrm{~K}}-\mathrm{PEG}_{5 \mathrm{~K}}$ nanoparticles (the particle size 
increased from $111.9 \pm 0.4$ to $141 \pm 1 \mathrm{~nm}$ as the FRR was increased from 0.1 to 0.8 ). Similar observations were reported by Donno et al (Donno et al., 2017).

\subsubsection{Total flow rate (TFR)}

The TFR is the sum of the flow rates of both solvent and antisolvent solutions in the microchip. Through TFR, it is possible to predict the residence time of the drug molecule within the microchip. Assuming that the cross-sectional area is constant, a higher TFR shortens the residence time of the drug molecule (or nuclei) inside the chip, and therefore reduces the risk of nanoparticle aggregates building up in the channel and the subsequent clogging of the chip (Amoyav and Benny, 2018; Wang et al., 2010).

During the preparation of hydrocortisone NPs in a microfluidic device, Ali et al (2009) observed that the particle size decreased from $289 \mathrm{~nm}$ to $80 \mathrm{~nm}$ by increasing the TFR from $1 \mathrm{ml} / \mathrm{min}$ to $3 \mathrm{ml} / \mathrm{min}$. Lababidi and co-workers observed a reduction in the mean diameter of PLGA NPs from 150 to $70 \mathrm{~nm}$ on increasing the TFR by increasing the flow rate of the external phase (keeping the FRR constant at 0.05 ) from 10 to $100 \mu \mathrm{L} / \mathrm{min}$ (Lababidi et al., 2019). Similar effects were reported in the manufacturing of PLGA NPs using acetonitrile as the solvent and Tris buffer at $\mathrm{pH} 7.4$ as antisolvent, where it was noticed that increasing TFR from 5 to $15 \mathrm{~mL} / \mathrm{min}$ led to a decrease in the mean particle diameter from about 380 to 125 nm (E. Chiesa et al., 2018).

A short residence time is favoured to produce small nanoparticles with a narrow particle size distribution as it lowers the possibility of heterogeneous nucleation resulting from contact between the supersaturated solution with the microchip walls. Furthermore, increasing the fluid flow speed inside the chip raises the Peclet number Pe (see Eq. 15) and hence increases 
the relative contribution of advection versus diffusion to the mass transfer (Saad and Prud'Homme, 2016; Tao et al., 2019).

\section{Microfluidic application in preparation of drug/polymer nanoparticles}

\subsection{The use of microfluidic systems to produce drug nanoparticles}

As previously mentioned, microfluidic systems have been used to produce drug nanosuspensions by nanoprecipitation method. In view of this application, we can categorize the used systems as mixers dominated either by diffusion or by turbulent mixing. Studies of drug particle nanosuspension production via diffusive micro-mixing have reported that the microchip path-length is too short to allow for sufficient mixing and the formation of stable precipitated particles (Aghajani et al., 2013; Ali et al., 2011, 2009; Zhao et al., 2007). Thus, the precipitation process in that case does not only depend on microchip geometry, FRR and TFR, but also depends on the vessel that receives the suspension after leaving the microchip. A mixing microdevice combined with sonication was reported by Ali and co-workers (Ali et al., 2011, 2009). The microchip outlet was subjected to dilution with stirring and exposed to sonication (unspecified intensity or time). Therefore, the influences of different microchip parameters in that case were masked by high-energy process (sonication) and by further dilution with mixing. Therefore often the properties of the particles generated using conventional flow-focusing droplet junction microchip may not be significantly different from the ones generated by conventional nanoprecipitation. However, with the advancement in chip design, microfluidics method can allow rapid diffusion and induce secondary flows like Taylor vortices or Dean vortices which can accelerate the mixing enormously (Capretto et al., 2013). 


\subsection{Bulk methods compared to microfluidic platforms for polymeric nanoparticles fabrication}

Microfluidic devices have been used in the literature to produce small nanoparticles with narrow size distribution explained by the efficient rapid mixing achieved by manipulating the mixing of the fluids in micro-channels (Karnik et al., 2008; Bally et al., 2012; Miladi et al., 2016). The polymer PLGA has been widely used to fabricate tuneable biodegradable nanoparticles in microfluidic devices. Xu and co-workers used a microfluidic flow-focusing method to manufacture doxorubicin- or tamoxifen-loaded PLGA nanoparticles (Xu et al., 2017). By changing the flow rate ratio and the polymer concentration they were able to precisely control the size of the PLGA nanoparticles (Xu et al., 2017). However, PLGA nanoparticles prepared using bulk nanoprecipitation methods can also be precisely controlled by tuning the processing parameters such as the polymer concentration or the solvent/antisolvent volume ratio (Wei Huang and Chenming Zhang, 2017).

Karnik and co-workers investigated the use of the microfluidics to fabricate smaller PLGAPEG nanoparticles compared to the bulk conventional methods (Bally et al., 2012; Miladi et al., 2016). The study reported an average particle size of $24 \mathrm{~nm}$ and $31 \mathrm{~nm}$ for the microfluidic nanoparticles and the conventional nanoparticles, respectively (Karnik et al., 2008). However, it's important to mention that ultra-small nanoparticles of $15 \mathrm{~nm}$ were fabricated by micropipette and shaking using the conventional nanoprecipitation technique (Reisch et al., 2015). These small nanoparticles were achieved by controlling the number of the charged group per polymer chain as one or two charged groups were found to significantly reduce the size of the nanoparticles (Reisch et al., 2015). This proves that when the processing parameters are precisely controlled, small nanoparticles can be achieved not only within microfluidic devices via enhanced mixing but also using conventional 
nanoprecipitation. But, in comparing the relative merits of the two approaches, it is important to consider carefully the functionality improvements (such as enhanced solubility) that are gained by size reduction in microfluidic devices. Such improvements might also be realised using bulk precipitation methods, which have the advantage of a substantially higher throughput rate and are easier to scale-up.

\subsection{Truth and myth: Limitations of microfluidics application in the production of nanoparticles}

\subsubsection{Productivity and scaling up}

According to the published data regarding the solute (drug/polymer) concentration used in microfluidic systems, it is clear that the production rate of the drug/polymeric nanoparticles is limited due to the microfluidic system dimensions and the used flow rates in comparison to conventional bulk mixing methods. For instance, Karnik and co-workers reported that the yield of the PLGA nanoparticles produced by a T-shaped hydrodynamic flow-focusing chip was 0.02376-0.792 g/day (Karnik et al., 2008). For drug nanosuspensions, using a Y-shaped microchip achieved the production of hydrocortisone nanosuspension at a throughput rate of $18 \mathrm{~g} /$ day (Ali et al., 2011). More examples and their nanoparticle production can be found in a previously published review (Ding et al., 2016).

The throughput can be increased by either raising the solute concentration (but not so much as to increase the size of the NPs) or increasing the TFR. Confined impinging jets mixers (CIJMs) and multi-inlet vortex mixers (MIVMs) have a much higher production rate (up to 864 g/day) than most other conventional microfluidic chips. However, increasing the flow rate into the range around $100 \mathrm{ml} / \mathrm{min}$ means that the channels must be able to tolerate and withstand the pressure (such as steel microchips) (Saad and Prud'Homme, 2016; Tao et al., 
2019). In these systems, the Peclet number $P e$ (Eq. 15) is high and the mixing will be dominated by advection.

Production parallelization is another approach to increase the productivity (Amoyav and Benny, 2018). Kang and co-workers were the first to report the manufacture of a parallel linemicrochip to produce polymeric nanoparticles. They used parallel mixing lines between water (the anti-solvent) and the polymer solution in a 100 outlet channel to increase the TFR to 6 $\mathrm{ml} / \mathrm{min}$ using a PDMS microchip (Kang et al., 2013). A parallel 3D hydrodynamic flowfocusing chip made of PDMS (Figure 16), and designed by Lim and the co-workers, was found to be efficient for the production of 13-150 nm PLGA-PEG nanoparticles. It had an enhanced throughput of $25 \mathrm{mg}$ of polymeric nanoparticles in $20 \mathrm{~min}$ compared to 5 hours when using single layer microchips (Lim et al., 2014a; Rhee et al., 2011). Although levelling up microfluidics into the industrial scale remains a challenge (even after parallelization), there are nonetheless promising developments in using microchips to increase nanoparticle production rates.

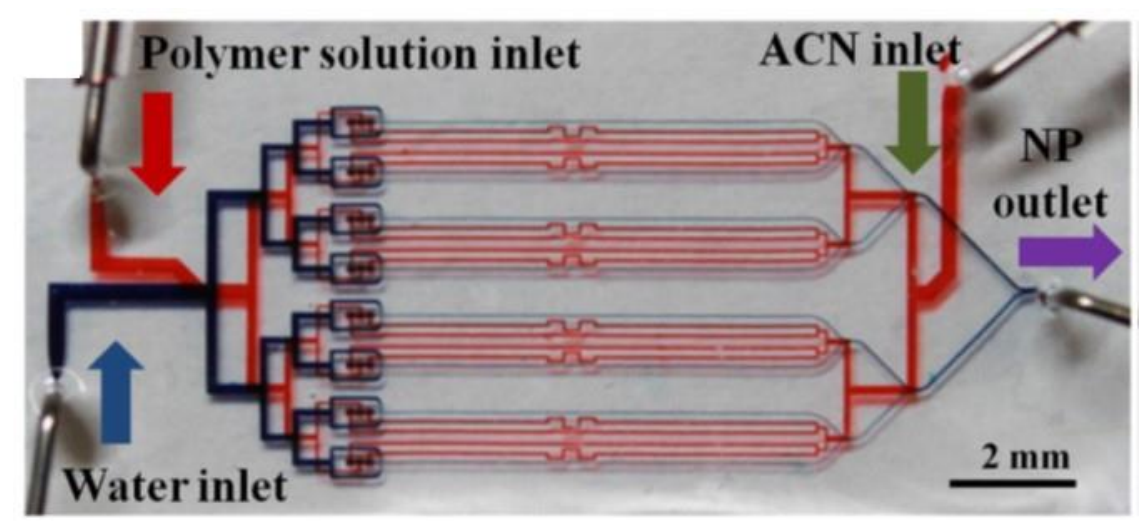

Figure 16. An example of a parallel 3D hydrodynamic flow-focusing microchip (reprinted with permission from (Lim et al., 2014a)).

\subsubsection{Chip clogging}


Clogging of microchips is another problem that makes it difficult to maintain a continuous process flow. Increasing the solute concentration or using relatively low TFR leads to precipitation in the channel and subsequent particle growth inside the chip. Eventually this results in the complete clogging of the microchannel. Surface interactions such as the aggregation of polymeric nanoparticles (e.g. PLGA NPs) on the inner hydrophobic surfaces of the PDMS chips is another possible cause of chips clogging (Rhee et al., 2011). To mitigate clogging 3D flow focusing microchips have been designed (Figure 17) to keep the precipitation of the nanoparticles away from the walls of the microchannels and thus allow the flow to be continued without any disruption or clogging. Different channel/microcapillary designs have been tested for this purpose and several promising studies reported the prevention of clogging and maintenance of flow continuity (Lim et al., 2014a, 2014b; Liu et al., 2017; Lorenz et al., 2018; Rhee et al., 2011).

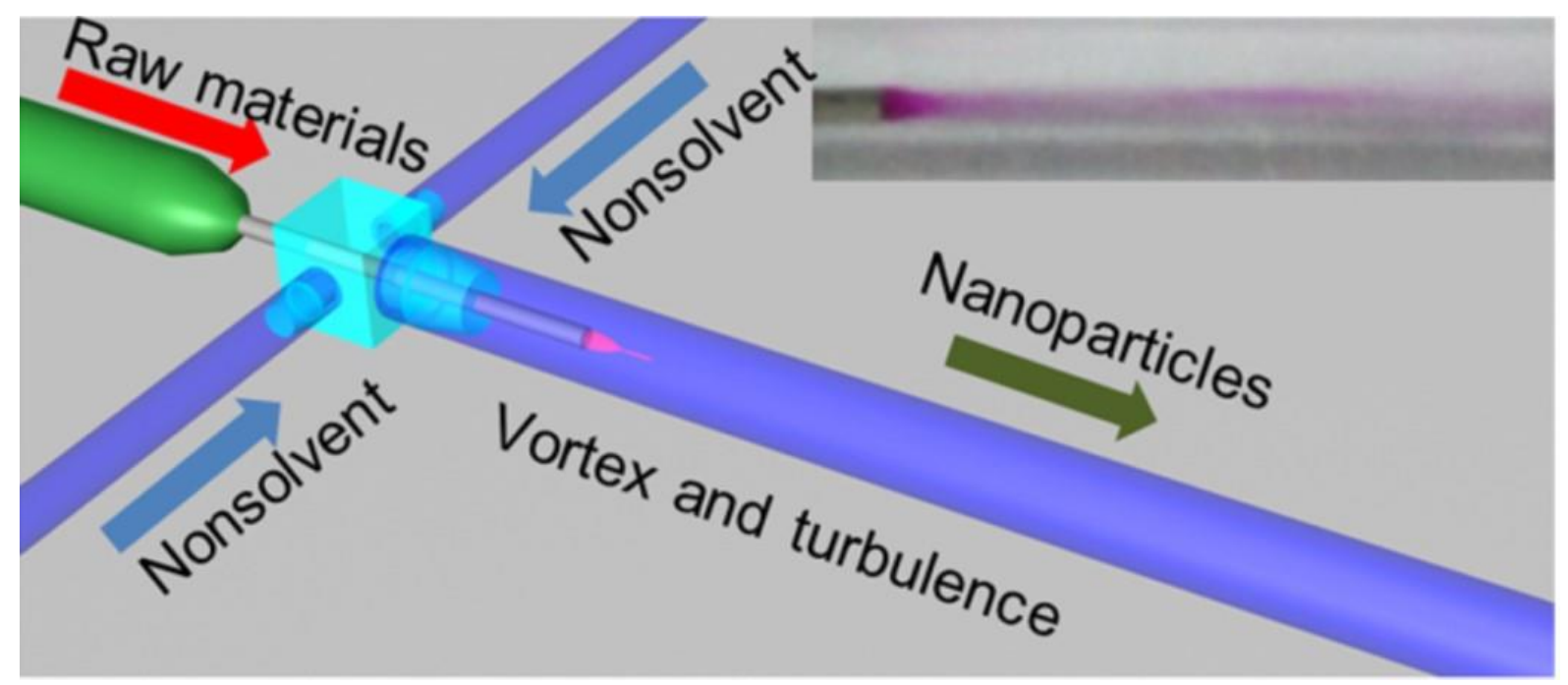

Figure 17. 3D hydrodynamic flow-focusing to prevent microchip clogging (reprinted with permission from (Lim et al., 2014b)).

\section{Conclusion}


Using precipitation as the mode of particle formation, microfluidic devices with miniaturemachined channels can achieve more precise control of the mixing of the fluid phases than conventional bulk mixing methods. In terms of drug-loaded nanoparticles, microfluidic methods allow tuneability to the size and drug loading which can often be difficult to achieve by conventional preparation methods. Most microfluidic methods still face challenges in scale-up to achieve the same throughput as conventional methods for large batch production, although there are promising reports of improvements, In practice, the feasibility of using microfluidics to generate particles, and to control the quality of the particles, is highly dependent on the materials used as well as the design of the microfluidic channel and the surface chemistry that affects the flow and mixing of the fluid phases. Although material and surface chemistry aspects have been well studied, there is still no systematic analysis of the correlation between particle properties and the microfluidic device design. More research is are needed before the widespread adoption of this processing technique for research and industrial applications can be realised.

\section{References:}

Aghajani, M., Shahverdi, A.R., Rezayat, S.M., Amini, M.A., Amani, A., 2013. Preparation and optimization of acetaminophen nanosuspension through nanoprecipitation using microfluidic devices: an artificial neural networks study. Pharm. Dev. Technol. 18, 609618. https://doi.org/10.3109/10837450.2011.649854

Ahn, J., Ko, J., Lee, S., Yu, J., Kim, Y.T., Jeon, N.L., 2018. Microfluidics in nanoparticle drug delivery; From synthesis to pre-clinical screening. Adv. Drug Deliv. Rev. 128, 2953. https://doi.org/10.1016/j.addr.2018.04.001

Ali, H.S.M., York, P., Ali, A.M.A., Blagden, N., 2011. Hydrocortisone nanosuspensions for ophthalmic delivery: A comparative study between microfluidic nanoprecipitation and wet milling. J. Control. Release 149, 175-181. https://doi.org/10.1016/j.jconrel.2010.10.007

Ali, H.S.M., York, P., Blagden, N., 2009. Preparation of hydrocortisone nanosuspension through a bottom-up nanoprecipitation technique using microfluidic reactors. Int. J. Pharm. 375, 107-113. https://doi.org/10.1016/j.ijpharm.2009.03.029

Allémann, E., Gurny, R., Doelker, E., 1992. Preparation of aqueous polymeric nanodispersions by a reversible salting-out process: influence of process parameters on 
particle size. Int. J. Pharm. 87, 247-253. https://doi.org/10.1016/0378-5173(92)90249-2

Amani, A., Ali, H., Blagden, N., York, P., 2014. Evaluation of a nanosuspension formulation prepared through microfluidic reactors for pulmonary delivery of budesonide using nebulizers. Iran. J. Pharm. Res. 13, 785-795. https://doi.org/10.22037/ijpr.2014.1537

Amoyav, B., Benny, O., 2018. Controlled and tunable polymer particles' production using a single microfluidic device. Appl. Nanosci. 8, 905-914. https://doi.org/10.1007/s13204018-0790-0

Baby, T., Liu, Y., Middelberg, A.P.J., Zhao, C.X., 2017. Fundamental studies on throughput capacities of hydrodynamic flow-focusing microfluidics for producing monodisperse polymer nanoparticles. Chem. Eng. Sci. 169, 128-139.

https://doi.org/10.1016/j.ces.2017.04.046

Bally, F., Garg, D.K., Serra, C.A., Hoarau, Y., Anton, N., Brochon, C., Parida, D., Vandamme, T., Hadziioannou, G., 2012. Improved size-tunable preparation of polymeric nanoparticles by microfluidic nanoprecipitation. Polymer (Guildf). 53, 50455051. https://doi.org/10.1016/j.polymer.2012.08.039

Beißner, N., Lorenz, T., Reichl, S., 2016. Organ on Chip, in: Dietzel, A. (Ed.), Microsystems for Pharmatechnology. Springer International Publishing, Cham, pp. 299-339.

Bhise, N.S., Ribas, J., Manoharan, V., Zhang, Y.S., Polini, A., Massa, S., Dokmeci, M.R., Khademhosseini, A., 2014. Organ-on-a-chip platforms for studying drug delivery systems. J. Control. Release 190, 82-93.

Budhian, A., Siegel, S.J., Winey, K.I., 2007. Haloperidol-loaded PLGA nanoparticles: Systematic study of particle size and drug content. Int. J. Pharm. 336, 367-375. https://doi.org/10.1016/j.ijpharm.2006.11.061

Cao, G., Wang, Y., 2011. Nanostructures and Nanomaterials Synthesis, Properties, and Applications, 2nd ed. World scientific.

Capretto, L., Carugo, D., Mazzitelli, S., Nastruzzi, C., Zhang, X., 2013. Microfluidic and labon-a-chip preparation routes for organic nanoparticles and vesicular systems for nanomedicine applications. Adv. Drug Deliv. Rev. 65, 1496-1532. https://doi.org/10.1016/j.addr.2013.08.002

Chan, H.K., Kwok, P.C.L., 2011. Production methods for nanodrug particles using the bottom-up approach. Adv. Drug Deliv. Rev. 63, 406-416. https://doi.org/10.1016/j.addr.2011.03.011

Cheng, J., Teply, B.A., Sherifi, I., Sung, J., Luther, G., Gu, F.X., Levy-Nissenbaum, E., Radovic-Moreno, A.F., Langer, R., Farokhzad, O.C., 2007. Formulation of functionalized PLGA-PEG nanoparticles for in vivo targeted drug delivery. Biomaterials 28, 869-876. https://doi.org/10.1016/j.biomaterials.2006.09.047

Chiesa, E., Dorati, R., Modena, T., Conti, B., Genta, I., 2018. Multivariate analysis for the optimization of microfluidics-assisted nanoprecipitation method intended for the loading of small hydrophilic drugs into PLGA nanoparticles. Int. J. Pharm. 536, 165-177. https://doi.org/10.1016/j.ijpharm.2017.11.044

Chiesa, Enrica, Dorati, R., Pisani, S., Conti, B., Bergamini, G., Modena, T., Genta, I., 2018. The Microfluidic Technique and the Manufacturing of Polysaccharide Nanoparticles. Pharmaceutics 10, 267. https://doi.org/10.3390/pharmaceutics10040267 
Chorny, M., Fishbein, I., Danenberg, H.D., Golomb, G., 2002. Lipophilic drug loaded nanospheres prepared by nanoprecipitation: Effect of formulation variables on size, drug recovery and release kinetics. J. Control. Release 83, 389-400. https://doi.org/10.1016/S0168-3659(02)00211-0

Dalvi, S. V., Dave, R.N., 2009. Controlling particle size of a poorly water-soluble drug using ultrasound and stabilizers in antisolvent precipitation. Ind. Eng. Chem. Res. 48, 75817593. https://doi.org/10.1021/ie900248f

Damiati, Samar, Kompella, U., Damiati, Safa, Kodzius, R., 2018. Microfluidic Devices for Drug Delivery Systems and Drug Screening. Genes (Basel). 9, 103. https://doi.org/10.3390/genes9020103

De Yoreo, J.J., 2003. Principles of Crystal Nucleation and Growth. Rev. Mineral. Geochemistry 54, 57-93. https://doi.org/10.2113/0540057

Dhumal, R.S., Biradar, S. V., Yamamura, S., Paradkar, A.R., York, P., 2008. Preparation of amorphous cefuroxime axetil nanoparticles by sonoprecipitation for enhancement of bioavailabilityDhumal, R. S., Biradar, S. V., Yamamura, S., Paradkar, A. R., \& York, P. (2008). Preparation of amorphous cefuroxime axetil nanoparticles by so. Eur. J. Pharm. Biopharm. 70, 109-115. https://doi.org/10.1016/j.ejpb.2008.04.001

Dietzel, A., 2016. A Brief Introduction to Microfluidics, in: Microsystems for Pharmatechnology. Springer International Publishing, Cham, pp. 1-21.

Dimo Kashchiev, 2000. Nucleation, 1st ed. Elsevier Science.

Ding, S., Anton, N., Vandamme, T.F., Serra, C.A., 2016. Microfluidic nanoprecipitation systems for preparing pure drug or polymeric drug loaded nanoparticles: an overview. Expert Opin. Drug Deliv. 13, 1447-1460. https://doi.org/10.1080/17425247.2016.1193151

Dong, Y., Feng, S.S., 2004. Methoxy poly(ethylene glycol)-poly(lactide) (MPEG-PLA) nanoparticles for controlled delivery of anticancer drugs. Biomaterials 25, 2843-2849. https://doi.org/10.1016/j.biomaterials.2003.09.055

Dong, Y., Ng, W.K., Hu, J., Shen, S., Tan, R.B.H., 2010. A continuous and highly effective static mixing process for antisolvent precipitation of nanoparticles of poorly watersoluble drugs. Int. J. Pharm. 386, 256-261. https://doi.org/10.1016/j.ijpharm.2009.11.007

Donno, R., Gennari, A., Lallana, E., De La Rosa, J.M.R., d'Arcy, R., Treacher, K., Hill, K., Ashford, M., Tirelli, N., 2017. Nanomanufacturing through microfluidic-assisted nanoprecipitation: Advanced analytics and structure-activity relationships. Int. J. Pharm. 534, 97-107. https://doi.org/10.1016/j.ijpharm.2017.10.006

Ealias, A.M., Saravanakumar, M.P., 2017. A review on the classification, characterisation, synthesis of nanoparticles and their application. IOP Conf. Ser. Mater. Sci. Eng. 263. https://doi.org/10.1088/1757-899X/263/3/032019

Ebert, S., Koo, C.K.W., Weiss, J., McClements, D.J., 2017. Continuous production of coreshell protein nanoparticles by antisolvent precipitation using dual-channel microfluidization: Caseinate-coated zein nanoparticles. Food Res. Int. 92, 48-55. https://doi.org/10.1016/j.foodres.2016.12.020

Erfle, P., Riewe, J., Bunjes, H., Dietzel, A., 2019. Stabilized production of lipid nanoparticles 
of tunable size in Taylor flow glass devices with high-surface-quality 3D microchannels. Micromachines 10. https://doi.org/10.3390/mi10040220

Feng, Q., Sun, J., Jiang, X., 2016. Microfluidics-mediated assembly of functional nanoparticles for cancer-related pharmaceutical applications. Nanoscale 8, 1243012443. https://doi.org/10.1039/C5NR07964K

Feng, Q., Zhang, L., Liu, C., Li, X., Hu, G., Sun, J., Jiang, X., 2015. Microfluidic based high throughput synthesis of lipid-polymer hybrid nanoparticles with tunable diameters. Biomicrofluidics 9. https://doi.org/10.1063/1.4922957

Freag, M.S., Elnaggar, Y.S.R., Abdallah, O.Y., 2013. Development of novel polymerstabilized diosmin nanosuspensions: In vitro appraisal and ex vivo permeation. Int. J. Pharm. 454, 462-471. https://doi.org/10.1016/j.ijpharm.2013.06.039

Gajera, B.Y., Shah, D.A., Dave, R.H., 2019. Development of an amorphous nanosuspension by sonoprecipitation-formulation and process optimization using design of experiment methodology. Int. J. Pharm. 559, 348-359. https://doi.org/10.1016/j.ijpharm.2019.01.054

Guhagarkar, S.A., Malshe, V.C., Devarajan, P. V, 2009. Nanoparticles of polyethylene sebacate: A new biodegradable polymer. AAPS PharmSciTech 10, 935-942. https://doi.org/10.1208/s12249-009-9284-4

Günther, A., Jensen, K.F., 2006. Multiphase microfluidics: From flow characteristics to chemical and materials synthesis. Lab Chip. https://doi.org/10.1039/b609851g

Gupta, R., Fletcher, D., Haynes, B., 2010. Taylor flow in microchannels: A review of experimental and computational work. J. Comput. Multiph. Flows 2, 1-31. https://doi.org/10.1260/1757-482X.2.1.1

Haberkorn, H., Franke, D., Frechen, T., Goesele, W., Rieger, J., 2003. Early stages of particle formation in precipitation reactions - Quinacridone and boehmite as generic examples. J. Colloid Interface Sci. 259, 112-126. https://doi.org/10.1016/S0021-9797(03)00024-9

Hessel, V., Löwe, H., Schönfeld, F., 2005. Micromixers - A review on passive and active mixing principles. Chem. Eng. Sci. 60, 2479-2501. https://doi.org/10.1016/j.ces.2004.11.033

Jeevanandam, J., Barhoum, A., Chan, Y.S., Dufresne, A., Danquah, M.K., 2018. Review on nanoparticles and nanostructured materials: History, sources, toxicity and regulations. Beilstein J. Nanotechnol. 9, 1050-1074. https://doi.org/10.3762/bjnano.9.98

Kakran, M., Sahoo, N.G., Li, L., Judeh, Z., 2012. Fabrication of quercetin nanoparticles by anti-solvent precipitation method for enhanced dissolution. Powder Technol. 223, 5964. https://doi.org/10.1016/j.powtec.2011.08.021

Kang, X., Luo, C., Wei, Q., Xiong, C., Chen, Q., Chen, Y., Ouyang, Q., 2013. Mass production of highly monodisperse polymeric nanoparticles by parallel flow focusing system. Microfluid. Nanofluidics 15, 337-345. https://doi.org/10.1007/s10404-013$1152-6$

Karakucuk, A., Teksin, Z.S., Eroglu, H., Celebi, N., 2019. Evaluation of improved oral bioavailability of ritonavir nanosuspension. Eur. J. Pharm. Sci. 131, 153-158. https://doi.org/10.1016/j.ejps.2019.02.028 
Karnik, R., Gu, F., Basto, P., Cannizzaro, C., Dean, L., Kyei-Manu, W., Langer, R., Farokhzad, O.C., 2008. Microfluidic platform for controlled synthesis of polymeric nanoparticles. Nano Lett. 8, 2906-2912. https://doi.org/10.1021/nl801736q

Kimura, H., Tomatsu, K., Saiki, H., Arimitsu, K., Ono, M., Kawashima, H., Iwata, R., Nakanishi, H., Ozeki, E., Kuge, Y., Saji, H., 2016. Continuous-flow synthesis of Nsuccinimidyl 4-[18F]fluorobenzoate using a single microfluidic chip. PLoS One 11, 115. https://doi.org/10.1371/journal.pone.0159303

Kulkarni, S.A., Myerson, A.S., 2017. Reversible control of solubility using functionalized nanoparticles. Chem. Commun. 53, 1429-1432. https://doi.org/10.1039/c6cc09390f

Lababidi, N., Sigal, V., Koenneke, A., Schwarzkopf, K., Manz, A., Schneider, M., 2019. Microfluidics as tool to prepare size-tunable PLGA nanoparticles with high curcumin encapsulation for efficient mucus penetration. Beilstein J. Nanotechnol. 10, 2280-2293. https://doi.org/10.3762/bjnano.10.220

Lamprecht, A., Arica, B., 2005. In Vitro Evaluation of Betamethasone-Loaded Nanoparticles. Drug Dev. Ind. Pharm. 31, 19-24. https://doi.org/10.1081/DDC-200043937

Li, X., Tian, T., 2018. Recent advances in an organ-on-a-chip: Biomarker analysis and applications. Anal. Methods 10, 3122-3130. https://doi.org/10.1039/c8ay00970h

Lim, J.-M., Bertrand, N., Valencia, P.M., Rhee, M., Langer, R., Jon, S., Farokhzad, O.C., Karnik, R., 2014a. Parallel microfluidic synthesis of size-tunable polymeric nanoparticles using 3D flow focusing towards in vivo study. Nanomedicine Nanotechnology, Biol. Med. 10, 401-409. https://doi.org/10.1016/j.nano.2013.08.003

Lim, J.-M., Swami, A., Gilson, L.M., Chopra, S., Choi, S., Wu, J., Langer, R., Karnik, R., Farokhzad, O.C., 2014b. Ultra-High Throughput Synthesis of Nanoparticles with Homogeneous Size Distribution Using a Coaxial Turbulent Jet Mixer. ACS Nano 8, 6056-6065. https://doi.org/10.1021/nn501371n

Limayem Blouza, I., Charcosset, C., Sfar, S., Fessi, H., 2006. Preparation and characterization of spironolactone-loaded nanocapsules for paediatric use. Int. J. Pharm. 325, 124-131. https://doi.org/10.1016/j.ijpharm.2006.06.022

Liu, D., Cito, S., Zhang, Y., Wang, C.F., Sikanen, T.M., Santos, H.A., 2015. A versatile and robust microfluidic platform toward high throughput synthesis of homogeneous nanoparticles with tunable properties. Adv. Mater. 27, 2298-2304. https://doi.org/10.1002/adma.201405408

Liu, D., Zhang, H., Cito, S., Fan, J., Mäkilä, E., Salonen, J., Hirvonen, J., Sikanen, T.M., Weitz, D.A., Santos, H.A., 2017. Core/Shell Nanocomposites Produced by Superfast Sequential Microfluidic Nanoprecipitation. Nano Lett. 17, 606-614. https://doi.org/10.1021/acs.nanolett.6b03251

Liu, D., Zhang, H., Fontana, F., Hirvonen, J.T., Santos, H.A., 2018. Current developments and applications of microfluidic technology toward clinical translation of nanomedicines. Adv. Drug Deliv. Rev. 128, 54-83. https://doi.org/10.1016/j.addr.2017.08.003

Lorenz, T., Bojko, S., Bunjes, H., Dietzel, A., 2018. An inert 3D emulsification device for individual precipitation and concentration of amorphous drug nanoparticles. Lab Chip 18, 627-638. https://doi.org/10.1039/C7LC01313B 
Lu, M., Ozcelik, A., Grigsby, C.L., Zhao, Y., Guo, F., Leong, K.W., Huang, T.J., 2016. Microfluidic hydrodynamic focusing for synthesis of nanomaterials. Nano Today 11, 778-792. https://doi.org/10.1016/j.nantod.2016.10.006

Marre, S., Jensen, K.F., 2010. Synthesis of micro and nanostructures in microfluidic systems. Chem. Soc. Rev. 39, 1183-1202. https://doi.org/10.1039/b821324k

Martínez Rivas, C.J., Tarhini, M., Badri, W., Miladi, K., Greige-Gerges, H., Nazari, Q.A., Galindo Rodríguez, S.A., Román, R.Á., Fessi, H., Elaissari, A., 2017. Nanoprecipitation process: From encapsulation to drug delivery. Int. J. Pharm. 532, 66-81. https://doi.org/10.1016/j.ijpharm.2017.08.064

Martins, J.P., Torrieri, G., Santos, H.A., 2018. The importance of microfluidics for the preparation of nanoparticles as advanced drug delivery systems. Expert Opin. Drug Deliv. 15, 469-479. https://doi.org/10.1080/17425247.2018.1446936

Miladi, K., Sfar, S., Fessi, H., Elaissari, A., 2016. Nanoprecipitation Process: From Particle Preparation to In Vivo Applications, in: Polymer Nanoparticles for Nanomedicines. Springer International Publishing, Cham, pp. 17-53. https://doi.org/10.1007/978-3-31941421-8_2

Mohammad, B.-J., Siahi, M.R., Nokhodchi, A., Hallaj, S., Hasan, M., Barzegar-Jalali, M., Adibkia, K., Valizadeh, H., Reza, M., Shadbad, S., Omidi, Y., Mohammadi, G., Nezhadi, S.H., 2008. Kinetic analysis of drug release from nanoparticles. J. Pharm. Pharm. Sci. 11, 167-177.

Nagy, Z.K., Fujiwara, M., Braatz, R.D., 2019. Monitoring and Advanced Control of Crystallization Processes, in: Handbook of Industrial Crystallization. pp. 313-345.

Nehilla, B.J., Bergkvist, M., Popat, K.C., Desai, T.A., 2008. Purified and surfactant-free coenzyme Q10-loaded biodegradable nanoparticles. Int. J. Pharm. 348, 107-114. https://doi.org/10.1016/j.ijpharm.2007.07.001

Nguyen, N.T., 2011. Micromixers: Fundamentals, Design and Fabrication, Micro and Nano Technologies. Elsevier Science.

Nguyen, N.T., Huang, X., 2006. Modelling, fabrication and characterization of a polymeric micromixer based on sequential segmentation. Biomed. Microdevices 8, 133-139. https://doi.org/10.1007/s10544-006-7708-4

Nguyen, N.T., Shaegh, S.A.M., Kashaninejad, N., Phan, D.T., 2013. Design, fabrication and characterization of drug delivery systems based on lab-on-a-chip technology. Adv. Drug Deliv. Rev. 65, 1403-1419.

Oddy, M.H., Santiago, J.G., Mikkelsen, J.C., 2001. Electrokinetic instability micromixing. Anal. Chem. 73, 5822-5832. https://doi.org/10.1021/ac0155411

Pan, L.J., Tu, J.W., Ma, H.T., Yang, Y.J., Tian, Z.Q., Pang, D.W., Zhang, Z.L., 2017. Controllable synthesis of nanocrystals in droplet reactors. Lab Chip 18, 41-56. https://doi.org/10.1039/c7lc00800g

Panagiotou, T., Mesite, S. V., Fisher, R.J., 2009. Production of norfloxacin nanosuspensions using microfluidics reaction technology through solvent/antisolvent crystallization. Ind. Eng. Chem. Res. 48, 1761-1771. https://doi.org/10.1021/ie800955t

Pande, M., Ashok Bhaskarwar, 2016. Nanoparticles: Preparation and Characterization. 
Momentum Press ${ }^{\circledR}$, LLC.

Polte, J., 2015. Fundamental growth principles of colloidal metal nanoparticles - a new perspective. CrystEngComm 17, 6809-6830. https://doi.org/10.1039/c5ce01014d

Probst, C., Schneider, S., Loskill, P., 2018. High-throughput organ-on-a-chip systems: Current status and remaining challenges. Curr. Opin. Biomed. Eng. 6, 33-41. https://doi.org/10.1016/j.cobme.2018.02.004

Rahimi, M., Valeh-e-Sheyda, P., Rashidi, H., 2017. Statistical optimization of curcumin nanosuspension through liquid anti-solvent precipitation (LASP) process in a microfluidic platform: Box-Behnken design approach. Korean J. Chem. Eng. 34, 30173027. https://doi.org/10.1007/s11814-017-0201-3

Reisch, A., Runser, A., Arntz, Y., Mély, Y., Klymchenko, A.S., 2015. Charge-controlled nanoprecipitation as a modular approach to ultrasmall polymer nanocarriers: Making bright and stable nanoparticles. ACS Nano 9, 5104-5116.

https://doi.org/10.1021/acsnano.5b00214

Rhee, M., Valencia, P.M., Rodriguez, M.I., Langer, R., Farokhzad, O.C., Karnik, R., 2011. Synthesis of size-tunable polymeric nanoparticles enabled by 3D hydrodynamic flow focusing in single-layer microchannels. Adv. Mater. 23, 79-83. https://doi.org/10.1002/adma.201004333

Riahi, R., Tamayol, A., Shaegh, S.A.M., Ghaemmaghami, A.M., Dokmeci, M.R., Khademshosseini, A., 2015. Microfluidics for advanced drug delivery systems. Curr. Opin. Chem. Eng. 7, 101-112. https://doi.org/10.1016/j.coche.2014.12.001

Richter, C., Stegemann, D., Vierheller, A., Gothsch, T., Finke, J.H., Kwade, A., MüllerGoymann, C.C., Dietzel, A., Büttgenbach, S., 2013. Innovative process chain for the development of wear resistant 3D metal microsystems. Microelectron. Eng. 110, 392397. https://doi.org/10.1016/j.mee.2013.02.078

Saad, W.S., Prud'Homme, R.K., 2016. Principles of nanoparticle formation by flash nanoprecipitation. Nano Today 11, 212-227. https://doi.org/10.1016/j.nantod.2016.04.006

Salazar, J., Müller, R.H., Möschwitzer, J.P., 2014. Combinative Particle Size Reduction Technologies for the Production of Drug Nanocrystals. J. Pharm. 2014, 1-14. https://doi.org/10.1155/2014/265754

Sanjay, S.T., Zhou, W., Dou, M., Tavakoli, H., Ma, L., Xu, F., Li, X.J., 2018. Recent advances of controlled drug delivery using microfluidic platforms. Adv. Drug Deliv. Rev. 128, 3-28. https://doi.org/10.1016/j.addr.2017.09.013

Schianti, J.N., Cerize, N.N.P., Oliveira, A.M., Derenzo, S., Gongora-Rubio, M.R., 2011. Rifampicin nanosuspension production using microfluidic device, in: Technical Proceedings of the 2011 NSTI Nanotechnology Conference and Expo, NSTI-Nanotech 2011.

Sear, R.P., 2006. Heterogeneous and homogeneous nucleation compared: Rapid nucleation on microscopic impurities. J. Phys. Chem. B 110, 4985-4989.

https://doi.org/10.1021/jp056377e

Seremeta, K.P., Chiappetta, D.A., Sosnik, A., 2013. Poly(e\{open\}-caprolactone), Eudragit® RS 100 and poly(e\{open\}-caprolactone)/Eudragit@ RS 100 blend submicron particles 
for the sustained release of the antiretroviral efavirenz. Colloids Surfaces B

Biointerfaces 102, 441-449. https://doi.org/10.1016/j.colsurfb.2012.06.038

Shariare, M.H., Altamimi, M.A., Marzan, A.L., Tabassum, R., Jahan, B., Reza, H.M., Rahman, M., Ahsan, G.U., Kazi, M., 2019. In vitro dissolution and bioavailability study of furosemide nanosuspension prepared using design of experiment (DoE). Saudi Pharm. J. 27, 96-105. https://doi.org/10.1016/j.jsps.2018.09.002

Sinha, B., Müller, R.H., Möschwitzer, J.P., 2013. Bottom-up approaches for preparing drug nanocrystals: Formulations and factors affecting particle size. Int. J. Pharm. 453, 126141. https://doi.org/10.1016/j.ijpharm.2013.01.019

Soon-Eng Ong , Sam Zhang, Hejun Du, Y.F., 2008. Fundamental principles and applications of microfluidic systems. Front. Biosci. 13, 2757-2773.

Su, Y.F., Kim, H., Kovenklioglu, S., Lee, W.Y., 2007. Continuous nanoparticle production by microfluidic-based emulsion, mixing and crystallization. J. Solid State Chem. 180, 2625-2629. https://doi.org/10.1016/j.jssc.2007.06.033

Suh, Y.K., Kang, S., 2010. A review on mixing in microfluidics. Micromachines 1, 82-111. https://doi.org/10.3390/mi1030082

Tao, J., Chow, S.F., Zheng, Y., 2019. Application of flash nanoprecipitation to fabricate poorly water-soluble drug nanoparticles. Acta Pharm. Sin. B 9, 4-18. https://doi.org/10.1016/j.apsb.2018.11.001

Thanh, N.T.K., Maclean, N., Mahiddine, S., 2014. Mechanisms of nucleation and growth of nanoparticles in solution. Chem. Rev. 114, 7610-7630. https://doi.org/10.1021/cr400544s

Thorat, A.A., Dalvi, S. V., 2012. Liquid antisolvent precipitation and stabilization of nanoparticles of poorly water soluble drugs in aqueous suspensions: Recent developments and future perspective. Chem. Eng. J. 181-182, 1-34. https://doi.org/10.1016/j.cej.2011.12.044

Tian, W.-C., Finehout, E., 2009. Introduction to Microfluidics, in: Microfluidics for Biological Applications. Springer US, Boston, MA, pp. 1-34. https://doi.org/10.1007/978-0-387-09480-9_1

Tsui, J.H., Lee, W., Pun, S.H., Kim, J., Kim, D.H., 2013. Microfluidics-assisted in vitro drug screening and carrier production. Adv. Drug Deliv. Rev. 65, 1575-1588. https://doi.org/10.1016/j.addr.2013.07.004

Valencia, P.M., Farokhzad, O.C., Karnik, R., Langer, R., 2012. Microfluidic technologies for accelerating the clinical translation of nanoparticles. Nat. Nanotechnol. 7, 623-629. https://doi.org/10.1038/nnano.2012.168

Van Der Woerd, M., Ferree, D., Pusey, M., 2003. The promise of macromolecular crystallization in microfluidic chips. J. Struct. Biol. 142, 180-187. https://doi.org/10.1016/S1047-8477(03)00049-2

Van Eerdenbrugh, B., Vermant, J., Martens, J.A., Froyen, L., Van Humbeeck, J., Augustijns, P., Van den Mooter, G., 2009. A screening study of surface stabilization during the production of drug nanocrystals. J. Pharm. Sci. 98, 2091-2103.

https://doi.org/10.1002/jps.21563 
Vekilov, P.G., 2010. Nucleation. Cryst. Growth Des. 10, 5007-5019.

Verma, S., Kumar, S., Gokhale, R., Burgess, D.J., 2011. Physical stability of nanosuspensions: Investigation of the role of stabilizers on Ostwald ripening. Int. J. Pharm. 406, 145-152. https://doi.org/10.1016/j.ijpharm.2010.12.027

Wang, J.X., Zhang, Q.X., Zhou, Y., Shao, L., Chen, J.F., 2010. Microfluidic synthesis of amorphous cefuroxime axetil nanoparticles with size-dependent and enhanced dissolution rate. Chem. Eng. J. 162, 844-851. https://doi.org/10.1016/j.cej.2010.06.022

Wang, Z., Chen, J.F., Le, Y., Shen, Z.G., Yun, J., 2007. Preparation of ultrafine beclomethasone dipropionate drug powder by antisolvent precipitation. Ind. Eng. Chem. Res. 46, 4839-4845. https://doi.org/10.1021/ie0615537

Wei Huang, Chenming Zhang, 2017. Tuning the size of poly (lactic-co-glycolic acid)(PLGA) nanoparticles fabricated by nanoprecipitation. Biotechnol. J. 13, 1-19. https://doi.org/10.1002/biot.201700203.Tuning

Weigl, B.H., Bardell, R.L., Cabrera, C.R., 2003. Lab-on-a-chip for drug development. Adv. Drug Deliv. Rev. 55, 349-377. https://doi.org/10.1016/S0169-409X(02)00223-5

Whitesides, G.M., 2006. The origins and the future of microfluidics. Nature 442, 368-373. https://doi.org/10.1038/nature05058

Xia, D., Quan, P., Piao, Hongze, Piao, Hongyu, Sun, S., Yin, Y., Cui, F., 2010. Preparation of stable nitrendipine nanosuspensions using the precipitation-ultrasonication method for enhancement of dissolution and oral bioavailability. Eur. J. Pharm. Sci. 40, 325-334. https://doi.org/10.1016/j.ejps.2010.04.006

Xiang Y. Liu, 2001. Generic mechanism of heterogeneous nucleation and molecular interfacial effects. Adv. Cryst. Growth Res. 42-61.

Xu, J., Zhang, S., MacHado, A., Lecommandoux, S., Sandre, O., Gu, F., Colin, A., 2017. Controllable Microfluidic Production of Drug-Loaded PLGA Nanoparticles Using Partially Water-Miscible Mixed Solvent Microdroplets as a Precursor. Sci. Rep. 7, 1-12. https://doi.org/10.1038/s41598-017-05184-5

Yang, Z., Matsumoto, S., Goto, H., Matsumoto, M., Maeda, R., 2001. Ultrasonic micromixer for microfluidic systems. Sensors Actuators, A Phys. 93, 266-272. https://doi.org/10.1016/S0924-4247(01)00654-9

Yu, Q., Wu, X., Zhu, Q., Wu, W., Chen, Z., Li, Y., Lu, Y., 2018. Enhanced transdermal delivery of meloxicam by nanocrystals: Preparation, in vitro and in vivo evaluation. Asian J. Pharm. Sci. 13, 518-526. https://doi.org/10.1016/j.ajps.2017.10.004

Zhang, J.Y., Shen, Z.G., Zhong, J., Hu, T.T., Chen, J.F., Ma, Z.Q., Yun, J., 2006. Preparation of amorphous cefuroxime axetil nanoparticles by controlled nanoprecipitation method without surfactants. Int. J. Pharm. 323, 153-160. https://doi.org/10.1016/j.ijpharm.2006.05.048

Zhang, Z.B., Shen, Z.G., Wang, J.X., Zhao, H., Chen, J.F., Yun, J., 2009. Nanonization of megestrol acetate by liquid precipitation. Ind. Eng. Chem. Res. 48, 8493-8499. https://doi.org/10.1021/ie900944y

Zhao, H., Wang, J.X., Wang, Q.A., Chen, J.F., Yun, J., 2007. Controlled liquid antisolvent precipitation of hydrophobic pharmaceutical nanoparticles in a MicroChannel reactor. 
Ind. Eng. Chem. Res. 46, 8229-8235. https://doi.org/10.1021/ie070498e

Zweers, M.L.T., Grijpma, D.W., Engbers, G.H.M., Feijen, J., 2003. The Preparation of Monodisperse Biodegradable Polyester Nanoparticles with a Controlled Size. J. Biomed. Mater. Res. - Part B Appl. Biomater. 66, 559-566. https://doi.org/10.1002/jbm.b.10046 